\title{
Simultaneous chiral resolution of two racemic compounds by preferential cocrystallization
}

Fuli Zhou ${ }^{\ddagger}$, Oleksii Shemchuk ${ }^{\ddagger}$, Maxime D. Charpentier, Chloé Matheys, Laurent Collard ${ }^{\ddagger}$, Joop H. ter Horst, Tom Leyssens*

\begin{abstract}
We tap into an unexplored area of preferential crystallization, being the first to develop simultaneous chiral resolution of two racemic compounds by preferential cocrystallization. We highlight how the two racemic compounds $R S$-mandelic acid (MAN) and RS-etiracetam (ETI) can be combined together as enantiospecific R-MAN:R-ETI and S-Man:S-ETI cocrystals forming a stable conglomerate system and subsequently develop a cyclic preferential crystallization allowing to simultaneous resolve both compounds. The developed process leads to excellent enantiopurity both for etiracetam (ee>98\%) and mandelic acid (ee 95\%) enantiomers.
\end{abstract}

\section{Introduction}

Homochirality is an intrinsic property of the building blocks of life such as carbohydrates (sugars, starch, and cellulose), amino acids (peptides, proteins) and nucleic acids. ${ }^{[1]}$ Metabolic and regulatory processes mediated by biological systems are therefore often sensitive to stereochemistry, ${ }^{[2]}$ with the two enantiomers of an active pharmaceutical compound potentially presenting significant differences in pharmacological properties ${ }^{[3]}$ as is the case for Naproxen with the S-enantiomer being used as a nonsteroidal anti-inflammatory drug whereas the R-enantiomer causes liver poisoning. ${ }^{[4]}$ Over the last decades substantial efforts were placed in developing processes leading to enantiopure compounds. ${ }^{[1,5]}$ The asymmetric synthesis (chiral pool, ${ }^{[6]}$ the use of chiral auxiliaries, ${ }^{[7]}$ or asymmetric catalysis ${ }^{[8]}$ ) approach results in production of a single enantiomer, but is not always feasible or in some cases not cost-effective. An alternative pathway is then to synthesize the racemic mixture, and resolve both enantiomers through chiral chromatography, ${ }^{[9]}$ kinetic resolution, ${ }^{[10]}$ diastereoisomeric salt formation, ${ }^{[11]}$ cocrystallization ${ }^{[12]}$ or preferential crystallization (PC). ${ }^{[13]}$ The main limitation of this latter is the requirement for the target compound to crystallize as a conglomerate - a physical mixture of mirror-image crystalline phases exhibiting symmetrical enantiomeric excesses. ${ }^{[13 a]}$ The statistics for a specific compound to crystallize as a conglomerate is observed to be $<10 \%{ }^{[14]}$ and crystal engineering tools are used to transform racemic compound systems into conglomerate ones, typically through salt, ${ }^{[15]}$ solvate, ${ }^{[16]}$ or more recently cocrystal formation with the use of achiral agents (acid, base, solvent or coformer). ${ }^{[17]}[18]$

Going beyond the use of achiral compounds, racemic carboxylic acids and racemic amines were successfully combined to form conglomerate salts. ${ }^{[19],[20]}$ Here we present a novel approach in which two racemic compounds combine into enantiospecific cocrystals forming a conglomerate that leads to simultaneous decoupling of both compounds (Scheme 1). We highpoint the strength of such an approach, being the first, to simultaneously chirally resolve both racemic compounds through a single preferential crystallization process, leading to excellent enantiopurity for all enantiomers dissociated from their cocrystals. 


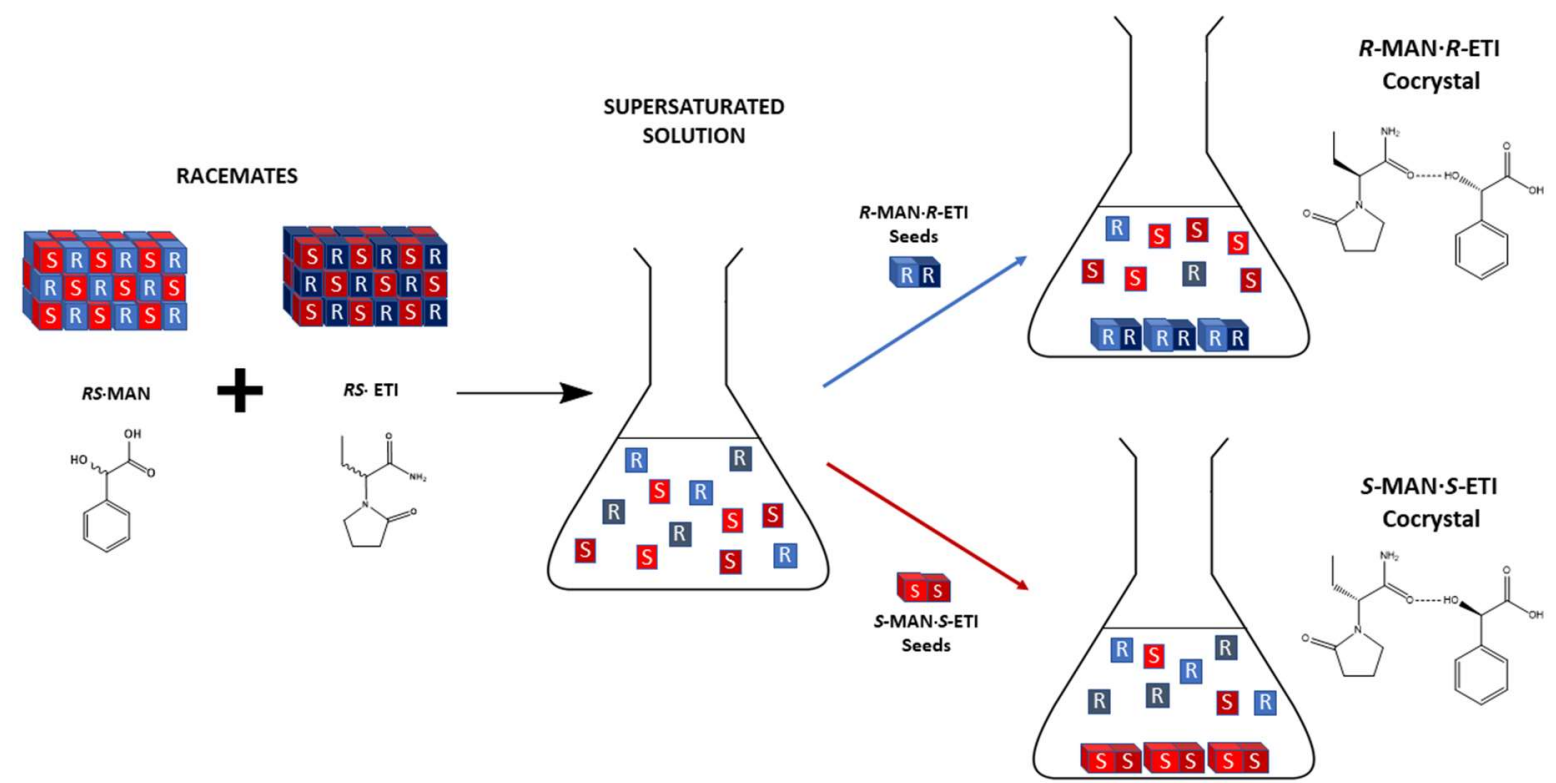

Scheme 1. Simultaneous resolution through preferential crystallization of both racemic compounds $R S$-mandelic acid and $R S$-etiracetam through formation of a conglomerate cocrystal system. Each cocrystal contains two chiral centers, with R-Man.R-ETI and S-MAN.S-ETI enantiomerically and not diastereomerically related.

\section{Results and discussions}

Neat grinding of an equimolar mixture of $R S$-mandelic acid (MAN) and RS-etiracetam (ETI) leads to the formation of a solid with an XRP-diffractogram overlapping that obtained when cocrystallizing $R$-mandelic acid and $R$-etiracetam (or $S$-mandelic acid and $S$-etiracetam). The pattern furthermore matches the simulated one from the S-MAN.S-ETI[21] cocrystal reported previously (CSD refcode YASGIK) ${ }^{[21]}$ (fig. 1). This finding indicates conglomerate formation, which incited us to gather further evidence confirming the existence of the $R-\mathrm{MAN} \cdot R-\mathrm{ETI} / S$ MAN-S-ETI conglomerate. Hand-picking a random single needle-shaped cocrystal obtained by slow evaporation of an initially undersaturated toluene solution containing a stoichiometric amount of RS-mandelic acid and RS-etiracetam showed both mandelic acid and etiracetam molecules inside the crystal to be of single handedness as illustrated by chiral HPLC (fig. 1) and $\mathrm{X}$-ray single crystal analysis.
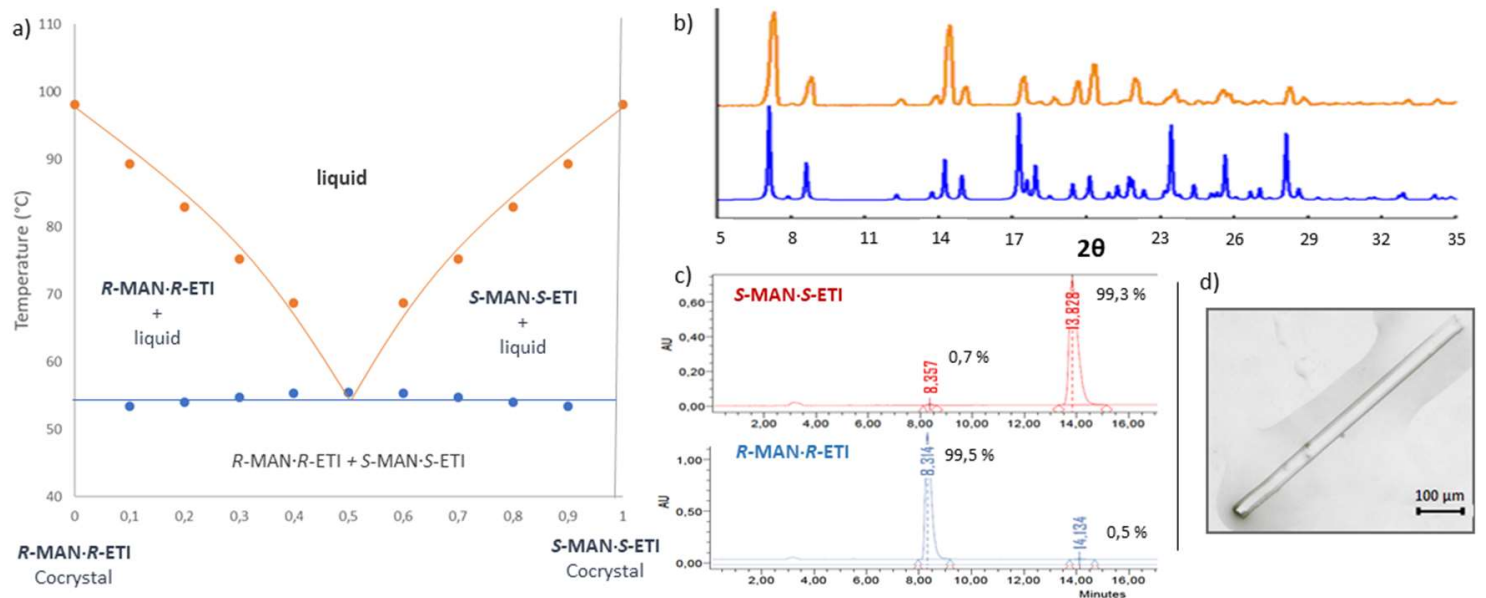
Fig. 1: a. Binary melting phase diagram of the $R$-MAN-R-ETI / S-MAN-S-ETI conglomerate; b. cHPLC chromatograms of randomly chosen single crystals yielding either one or the other enantiomer of etiracetam; $c$. Comparison of the experimental XRP-diffractogram of the mechanochemical cocrystallization between RSetiracetam and RS-mandelic acid (red) with the calculated pattern of S-MAN.S-ETI (blue; CSD refcode YASGIK ${ }^{[21]}$ ); d. An enantiopure cocrystal.

Thermodynamics studies confirm stable conglomerate formation between the enantiospecific cocrystals as illustrated by the experimental binary phase diagram (fig. 1 ; Table SI-2) showing a single eutectic equilibrium between the two symmetrical solid phases. This behavior highlights a complete dissociation of the racemic compounds into the more stable cocrystals when mixing equimolar ratios under equilibrated conditions.

Combining two racemic compounds to form a conglomerate, has the further ground-breaking advantage that not one but two compounds are simultaneously resolved at the solid state. We tapped into this resolution potential by developing a single preferential crystallization process, ${ }^{[13]}$ which allows for the simultaneous resolution of both compounds. To do so, we selected $^{[21 \mathrm{a}, 21 \mathrm{~b}]}$ a cyclic seeded isothermal preferential crystallization (SIPC) set-up. ${ }^{[22]}$ Selecting a suitable solvent is key as in the case of a cocrystallization based preferential crystallization, ${ }^{[18 a, 18 b]}$ one not only requires a sufficiently large meta-stable zone for crystallization but also needs to battle the inherent risk of one of the parent compounds crystallizing out separately or in combination with the cocrystal. Out of the 13 solvents tested (table SI-1), toluene and cyclohexane were shown to lead to suitable systems (see SI), with the former selected for further development.

As a strong increase in conglomerate solubility with temperature is observed beyond $27^{\circ} \mathrm{C}$ (Fig. SI-2), an equilibration temperature of $27^{\circ} \mathrm{C}$ was chosen to develop a seeded isothermal preferential crystallization process (SIPC), explained using a quasi-ternary phase diagram (experimental compositions given in the $\mathrm{SI}$ ). An initially saturated solution is created by recovering the supernatant of a conglomerate suspension at $30^{\circ} \mathrm{C}$ ( solubility $c^{*}=4 \% \mathrm{w} / \mathrm{w}$ ), and cooling this filtrate to $27^{\circ} \mathrm{C}\left(c^{*}=2.8 \% \mathrm{w} / \mathrm{w}\right)$ hereby obtaining a supersaturated solution shown by the blue square in Figure 3. Seeding this solution with S-MAN-S-ETI enantiopure cocrystal (eutomer) leads to its preferential crystallization and the solution composition ideally evolves towards the solubility line of this enantiopure material (point A). 


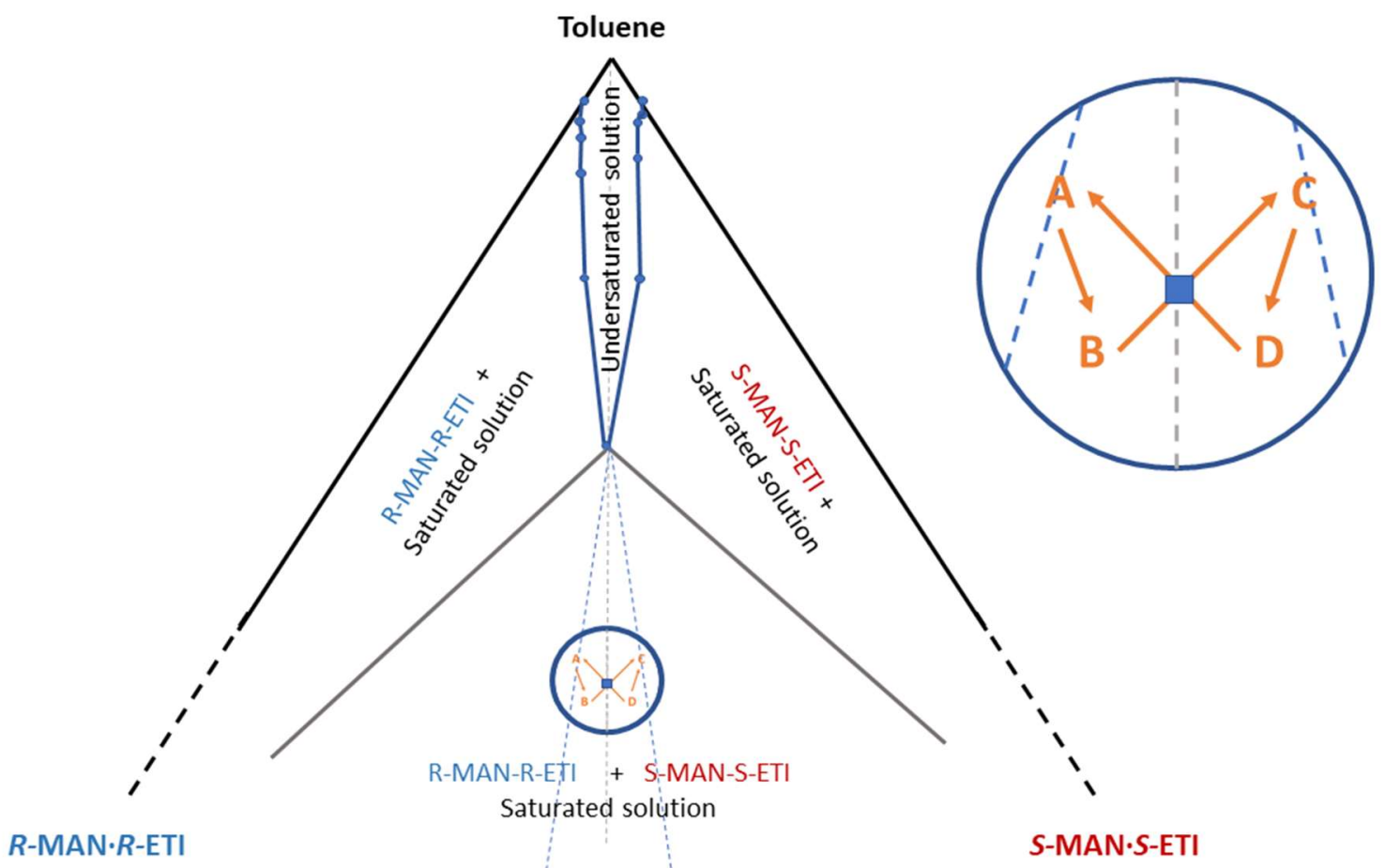

Fig. 3. Ternary phase diagram of the conglomerate system between cocrystals with SIPC procedure steps allowing resolution of both chiral systems.

As during this process, the solution remains supersaturated with respect to the $R$-MAN $R$-ETI distomer cocrystal, this latter can spontaneously crystallize out, rendering the preferential crystallization ineffective. It is therefore common to balance large yield and filter prior to reaching point $\mathrm{A}$ to reduce the likelihood of the spontaneous crystallization of the $R$-MAN $R$ ETI distomer cocrystal.

To pinpoint the time-frame in which spontaneous crystallization of the distomer cocrystal occurs, crystallization kinetics can be studied following the ee of both liquid and solid phases over time (Figure $4+\mathrm{SI}$ ). Upon seeding the supersaturated solution with $R-\mathrm{MAN} \cdot R$-ETI, the enantiomeric excess $e e$ in the solution initially increases as the solution depletes in $\mathrm{R}$ enantiomers through preferential crystallization of the $R$-MAN.R-ETI cocrystal, with the increasing mass of solids remaining enantiopure (Zone I). After about 35 minutes into the process, spontaneous nucleation of the symmetrical cocrystal (S-MAN-S-ETI) occurs, as shown by a decrease of both solution and solid ee (Zone II), with an establishment of a racemic composition in the solution after about $70 \mathrm{~min}$ (Zone III). Due to the excess of $R$-MAN.R-ETI cocrystal seeds in the overall composition a solid composition with a slight enantiomeric excess remains beyond $70 \mathrm{~min}$. 


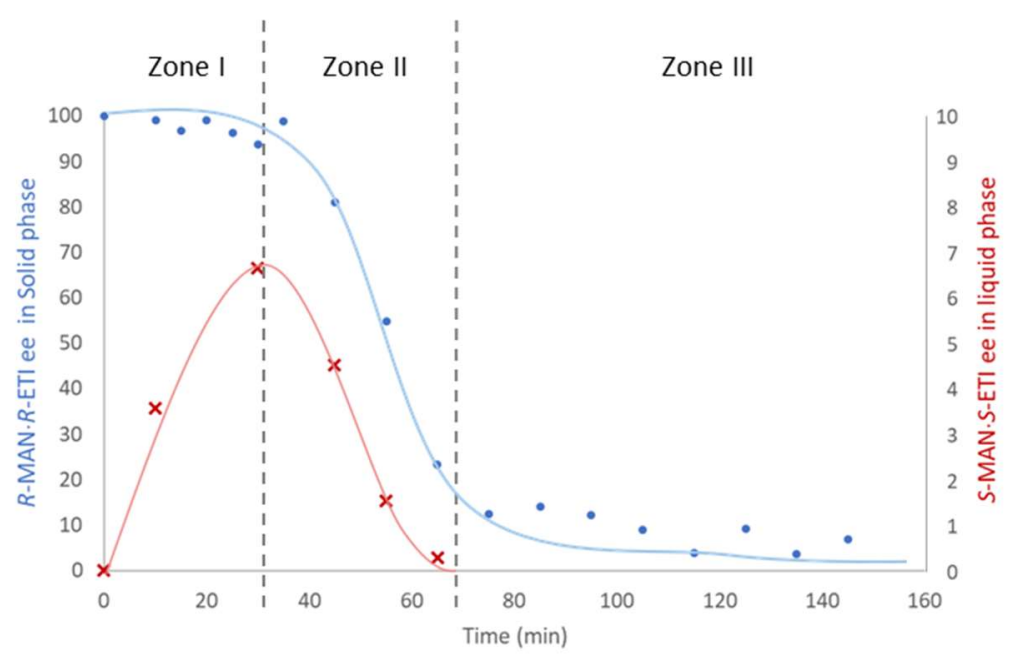

Fig. 4. Solution (blue) and solid (red) enantiomeric excess ee when seeding a supersaturated equimolar solution of $R S$-ETI and $R S$-MAN with $R$-MAN· $R$-ETI. Solution ee is given as a function of the $S$-enantiomers, whereas solid $e e$ is given as a function of the $R$-enantiomers.

This follow-up allows establishing conditions for a cyclic SIPC process, imposing filtration of the suspension containing only the desired cocrystal around 30 minutes into the process, prior to nucleation of the undesired one. Using this filtration time, a 6-cycle crystallization process was set-up as shown in table 1 and Figure 3 . The first cycle is different from all following cycles and starts with a supersaturated solution (c=35 mg. $\mathrm{ml}^{-1} ; \mathrm{c}^{*}=21 \mathrm{mg} \cdot \mathrm{ml}^{-1}$ ) as shown by the blue square. The supersaturated solution was obtained as the filtered supernatant of a $1.7 \mathrm{~g}$ racemic etiracetam and $1.52 \mathrm{~g}$ racemic mandelic acid suspension in 85 $\mathrm{g}$ of toluene at $30^{\circ} \mathrm{C}$. Seeding with S-MAN.S-ETI $(100 \mathrm{mg})$ leads to the preferential crystallization of this latter, with the suspension obtained after 30 minutes filtered, and recovery of a mother liquor (I) with composition shown by point A. $152 \mathrm{mg}$ of solid is recovered which means that besides the $100 \mathrm{mg}$ added seed $52 \mathrm{mg}$ of enantiopure material crystallized. The second cycle then starts by adding an equimolar amount in all enantiomers identical to the weight of enantiopure material recovered in the previous cycle $(52 \mathrm{mg})$ bringing the overall composition to point $\mathrm{B}$. To assure full dissolution prior to seeding, the solution was temporarily heated to $35^{\circ} \mathrm{C}$ and brought back to $27^{\circ} \mathrm{C}$. Seeding with homochiral seeds of opposite chirality with respect to the previous cycle, leads to preferential crystallization of this latter. The second cycle is completed by filtration of the suspension, leading to enantiopure material and a filtrate of composition $\mathrm{C}$. The third and further cycles are identical to cycle 2 , with the third cycle starting by addition of an equimolar amount in all enantiomers to bring the overall composition to D. Overall paired cycles (2-4-6) run from $A$ to $B$ to $C$, and unpaired cycles (3-5) from $C$ to $D$ to $A$.

Table 1. Cyclic SIPC of RS-MAN and RS-ETI. Mass of racemate added at the onset of each step ( $\mathrm{m}_{\mathrm{f}}$ ), mass of seed material added $\left(m_{s}\right)$, mass in enantiopure material recovered $\left(m_{r}\right)$, and ee of the resulting solids. Results for $S$ MAN.S-ETI are given in blue, for $R$-MAN $R$-ETI in red.

\begin{tabular}{cccccc}
\hline Cycle & $\mathrm{m}_{\mathrm{f}}(\mathrm{mg})$ & $\mathrm{m}_{\mathrm{s}}(\mathrm{mg})$ & $\mathrm{m}_{\mathrm{r}}(\mathrm{mg})$ & $e e_{\text {ETI }}(\%)$ & $e e_{\text {MAN }}(\%)$ \\
\hline 1 & $/$ & 100 & 152 & 98.0 & 95.7
\end{tabular}




\begin{tabular}{llllll}
2 & 52 & 100 & 200 & 98.3 & 93.7 \\
3 & 100 & 100 & 262 & 98.8 & 94.1 \\
4 & 162 & 100 & 272 & 99.0 & 94.8 \\
5 & 172 & 100 & 222 & 98.1 & 94.4 \\
6 & 122 & 100 & 256 & 98.1 & 94.7 \\
\hline
\end{tabular}

Table 1 shows the striking resolution power towards both compounds with enantiopure material reached at each step, alternating between both cocrystals. Spontaneous crystallization of the symmetrical cocrystal did not occur during any of the 6 cycles. Addition of equimolar amount in all enantiomers at the onset of cycles 2 to 6 led to the solutions with a slight initial ee, explaining the increased yield with respect to cycle 1 . Overall, we were able to resolve about $0.8 \mathrm{~g}$ of an equimolar mixture in all enantiomers through this small-scale process. This cycled SIPC experiment highlights the feasibility of simultaneous multiple successive entrainment steps adding a feed containing equimolar amounts of both racemic compounds between each step.

After combining pure enantiomers of a given chirality, cocrystal dissociation can be achieved to recover enantiopure mandelic acid and etiracetam compounds (see $\mathrm{SI}$ ). This allows recovering the materials and re-using them in subsequent cycles if needed. Overall, we showed a very potent resolution technique allowing for the simultaneous resolution of two racemic compounds using preferential crystallization. We are convinced that this contribution will incite people to investigate the potential of combining two racemic compounds for the formation of conglomerates, with an increasing future potential of seeing these processes being applied on industrial level.

\section{Conclusion}

In this contribution, we incite a change in mindset when trying to transform a racemic compound into a conglomerate through multi-component crystallization. We are the first to show how two racemic compounds can be combined as cocrystals with the two racemic compounds mandelic acid and etiracetam both being resolved simultaneously at the solid state. We then pioneer in showing how such a system results in simultaneous resolution of both compounds through a preferential crystallization process, obtaining excellent enantiopurity for both compounds using a cyclic preferential cocrystallization process.

\section{Acknowledgments}

FZ would like to thank the China Scholarship Council (CSC) for financial support. The "FSR Incoming Post-doctoral Fellowship" is acknowledged for financial support of OS. This work was financed by PDR T.0149.19. MDC and JHtH received funding as part of the CORE project (October 2016-September 2020) from the European Union Horizon 2020 Research and Innovation Program under the Marie Sklodowska-Curie grant arrangement No 722456 CORE ITN. 


\section{References:}

[1] N. M. Maier, P. Franco, W. Lindner, J. Chromatogr. A 2001, 906, 3-33.

[2] M. Lammerhofer, J. Chromatogr. A 2010, 1217, 814-856.

[3] R. Xie, L. Y. Chu, J. G. Deng, Chem. Soc. Rev. 2008, 37, 1243-1263.

[4] aS. Ahuja, Chiral Separation Methods for Pharmaceutical and Biotechnological Products, 2010; bW. F. Kean, C. J. Lock, H. E. Howard-Lock, Lancet 1991, 338, 1565-1568.

[5] a) G.-Q. Lin, Q.-D. You, J.-F. Cheng, Chiral drugs: Chemistry and biological action, John Wiley \& Sons, 2011; b) W. H. Brooks, W. C. Guida, K. G. Daniel, Curr. Top. Med. Chem. 2011, 11, 760770.

[6] H. U. Blaser, Chem. Rev. 1992, 92, 935-952.

[7] D. J. Ager, I. Prakash, D. R. Schaad, Chem. Rev. 1996, 96, 835-876.

[8] R. Noyori, Angew. Chem. Int. Ed. 2002, 41, 2008-2022.

[9] T. E. Beesley, R. P. Scott, Chiral chromatography, John Wiley \& Sons, 1999.

[10] C. S. Chen, Y. Fujimoto, G. Girdaukas, C. J. Sih, J. Am. Chem. Soc. 1982, 104, 7294-7299.

[11] D. Kozma, CRC handbook of optical resolutions via diastereomeric salt formation, Crc Press, 2001.

[12] a) G. Springuel, T. Leyssens, Cryst. Growth Des. 2012, 12, 3374-3378; b) B. Harmsen, T. Leyssens, Cryst. Growth Des. 2017, 18, 441-448.

[13] a) G. Coquerel, in Novel optical resolution technologies, Springer, 2006, pp. 1-51; b) G. Levilain, G. Coquerel, CrystEngComm 2010, 12, 1983-1992.

[14] a) S. Srisanga, J. H. ter Horst, Cryst. Growth Des. 2010, 10, 1808-1812; b) K. Sakai, N. Hirayama, R. Tamura, Novel optical resolution technologies, Vol. 269, Springer, 2007.

[15] W. W. Li, L. Spix, S. C. De Reus, H. Meekes, H. J. Kramer, E. Vlieg, J. H. ter Horst, Cryst. Growth Des. 2016, 16, 5563-5570.

[16] S. Wacharine-Antar, G. Levilain, V. Dupray, G. Coquerel, Org. Process Res. Dev. 2010, 14, 13581363.

[17] a) M. Guillot, J. de Meester, S. Huynen, L. Collard, K. Robeyns, O. Riant, T. Leyssens, Angew. Chem. Int. Ed. 2020; b) N. Blagden, M. de Matas, P. T. Gavan, P. York, Adv Drug Deliv Rev 2007, 59, 617-630; c) T. Friščić, W. Jones, J. Pharm. Pharmacol. 2010, 62, 1547-1559; d) C. C. Sun, Expert Opin Drug Deliv 2013, 10, 201-213.

[18] a) L. C. Harfouche, C. Brandel, Y. Cartigny, S. Petit, G. Coquerel, Chem. Eng. Technol. 2020, 43, 1093-1098; b) X. Buol, C. Caro Garrido, K. Robeyns, N. Tumanov, L. Collard, J. Wouters, T. Leyssens, Cryst. Growth Des. 2020, 20, 7979-7988; c) O. Shemchuk, L. Song, N. Tumanov, J. Wouters, D. Braga, F. Grepioni, T. Leyssens, Cryst. Growth Des. 2020, 20, 2602-2607.

[19] K. Kinbara, Y. Tagawa, K. Saigo, Tetrahedron-Asymmetry 2001, 12, 2927-2930.

[20] Y. Imai, K. Kawaguchi, N. Tajima, T. Sato, R. Kuroda, Y. Matsubara, Chem. Commun. 2008, 362364.

[21] G. Springuel, B. Norberg, K. Robeyns, J. Wouters, T. Leyssens, Cryst. Growth Des. 2011, 12, 475-484.

[22] a) C. Rougeot, J. E. Hein, Org. Process Res. Dev. 2015, 19, 1809-1819; b) M. P. Elsner, D. Fernandez Menendez, E. A. Muslera, A. Seidel-Morgenstern, Chirality 2005, 17 Suppl, S183195. 


\section{References:}

[1] B. Harmsen, T. Leyssens, Cryst. Growth Des. 2017, 18, 441-448.

[2] G. Springuel, B. Norberg, K. Robeyns, J. Wouters, T. Leyssens, Cryst. Growth Des. 2011, $12,475-484$.

[3] M. Klussmann, A. J. White, A. Armstrong, D. G. Blackmond, Angew. Chem. Int. Ed. 2006, 45, 7985-7989.

[4] J. Song, K.-X. Lou, X.-J. Li, X.-P. Wu, R.-X. Feng, Acta Crystallogr. Sect. Sect. E: Struct. Rep. Online 2003, 59, o1772-01773.

[5] A. O. Patil, W. T. Pennington, I. C. Paul, D. Y. Curtin, C. E. Dykstra, J. Am. Chem. Soc. 1987, 109, 1529-1535. 


\section{Supplementary Information}

\section{Simultaneous chiral resolution of two racemic compounds by preferential cocrystallization}

Fuli Zhou ${ }^{\ddagger}$, Oleksii Shemchuk ${ }^{\ddagger}$, Maxime D. Charpentier, Chloé Matheys, Laurent Collard ${ }^{\ddagger}$, Joop H. ter Horst, Tom Leyssens*

\section{Table of Contents}

page 2

1. Materials

page 2

2. Synthesis of R-MAN-R-ETI / S-MAN-S-ETI cocrystals

page 2

3. Congruency Experiments

page 2

4. Preparation of enantiopure seeds

page 3

5. X-ray Diffraction from Powder

page 3

6. Differential Scanning Calorimetry

page 3

7. Solubility curves

page 8

8. Quasi-Ternary isothermal phase diagram

page 10

9. Chiral High Performance Liquid Chromatography (cHPLC)

page 11

10. Preferential Crystallization Process

page 11

11. Liquid and solid phase sampling

page 16

12. Cocrystal separation in components

page 24

References

page 26 


\section{Experimental procedure.}

\section{$1 \quad$ Materials}

(S)-2-(2-oxopyrrolidin-1-yl)butanamide (Levetiracetam, S-ETI) was purchased from Xiamen Top Health Biochem Tech. Co., Ltd. (RS)-2-(2-oxopyrrolidin-1-yl)butanamide (RS-ETI) was prepared by racemization of (S)-2-(2-oxopyrrolidin-1-yl)butanamide. $10 \mathrm{~g}$ of (S)-2-(2oxopyrrolidin-1-yl)butanamide together with catalytic amount (0.05 eq.) of MeONa were added to $10 \mathrm{~mL}$ of $\mathrm{MeOH}$. The solution was kept at reflux under continuous stirring for $24 \mathrm{~h}$, and then cooled to room temperature. The compound crystallizes spontaneously. After filtration, the compound was washed twice with $\mathrm{MeOH}$. The recovered compound was used as such. $R$-ETI was obtained following the procedure previously described by our research group. ${ }^{[1]}$ RS-ETI (25g) was cocrystallized with $R$-mandelic acid (33.93g) in $150 \mathrm{~mL}$ of acetonitrile. The solution was seeded and left at $-10^{\circ} \mathrm{C}$ for 10 days. After filtration, the obtained solid material ( $R$-ETI-R-MAN) was suspended in diethyl ether $(500 \mathrm{~mL})$ for $24 \mathrm{~h}$. The suspension was filtered, and the recovered $R$-ETI solid analyzed by ${ }^{1} \mathrm{H}-\mathrm{NMR}$ (to confirm the absence of $R$-mandelic acid) and by chiral HPLC (to check the enantiopurity of $R$-ETI, >99\%).

All solvents used are commercially available from VWR. Solvents were used as received without any additional purification. (RS)-Mandelic acid (RS-MAN, >99\%), (S)-Mandelic acid (SMAN, >99\%) and (R)-Mandelic acid (R-MAN, >99\%) were purchased respectively from ACROS, $\mathrm{TCl}$ and Sigma-Aldrich and used as received.

\section{Mechanochemical synthesis of $R$-MAN $\cdot R$-ETI / S-MAN-S-ETI cocrystals}

The mechanochemical synthesis of the conglomerate cocrystal was performed by neat grinding, using a "Retsch Mixer Mill MM 400" equipped with two grinding jars containing five Eppendorf tubes of $2 \mathrm{~mL}$ each. Each tube was filled with an equimolar amount RS-etiracetam and RS-Mandelic acid and two $\varnothing 3 \mathrm{~mm}$ stainless-steel beads. Grinding was then performed at $30 \mathrm{~Hz}$ for 90 minutes.

\section{$3 \quad$ Congruency testing}

Congruency testing was performed slurrying equimolar suspensions $(0.5 \mathrm{~mol})$ of $R S$ etiracetam and RS-Mandelic acid in various solvents (table SI-1). The suspensions were stirred at $700 \mathrm{rpm}$ during 3 days at $25^{\circ} \mathrm{C}$ in sealed vials using a Cooling Thermomixer HLC. After $2 \mathrm{~h}$ of 
stirring, each vial is seeded with all possible solid forms (parent compounds and cocrystal conglomerate). Once the equilibrium reached, the powders were filtered, washed, dried, and analyzed using XRPD (table SI-1).

Table SI-1. Congruency testing of RS-etiracetam / RS-mandelic acid racemate in various solvents.

\begin{tabular}{lc}
\hline \multicolumn{1}{c}{ SOLVENT } & OUTCOME \\
\hline Ethanol & $R S$-ETI \\
Methanol & $R S$-ETI \\
Acetonitrile & $R S$-ETI \\
Acetone & $R S$-ETI \\
THF & $R S$-ETI \\
1-Propanol & $R S$-ETI \\
Isopropanol & $R S$-ETI \\
Ethyl acetate & $R S$-ETI \\
Methyl acetate & $R S$-ETI \\
Isopropyl acetate & $R S$-ETI \\
2-Butanol & $R S$-ETI \\
Toluene & $R$-MAN·R-ETI /S-MAN-S-ETI \\
Cyclohexane & $R$-MAN·R-ETI /S-MAN-S-ETI \\
\hline
\end{tabular}

\section{$4 \quad$ Preparation of enantiopure seeds}

Enantiopure seeds of R-MAN.R-ETI (S-MAN.S-ETI) were prepared according to the method described above (point 1). RS-ETI (2.5g) is slurried with R-MAN (S-MAN) (3.4g) in $15 \mathrm{ml}$ of acetonitrile at $-10^{\circ} \mathrm{C}$ for various days. The suspension is filtered, washed and the powder analyzed prior to being used as seed material.

\section{$5 \quad$ X-ray Diffraction from Powder}

For phase identification purposes X-ray powder diffraction (XRPD) patterns were collected on a PANalytical Bragg-Brentano diffractometer, using Ni-filtered CuK $\alpha(\lambda=1.54179 \AA$ ) with a $X^{\prime}$ Celerator detector in $4-40^{\circ} 2 \theta$ range (step size $0.0167^{\circ}, 45 \mathrm{kV} * 30 \mathrm{~mA}$ ).

\section{Differential Scanning Calorimetry}

Differential Scanning Calorimetry (DSC) experiments were performed from 25 to $120^{\circ} \mathrm{C}$, with a scanning rate of $10^{\circ} \mathrm{C} \cdot \mathrm{min}^{-1}$ on a "Mettler Toledo DSC $821^{\mathrm{e}}$. The solid phases were weighed (5-7 $\mathrm{mg}$ ) in aluminum pans with perforated lids. The purge gas was nitrogen, with a flow rate of $50 \mathrm{~mL} \cdot \mathrm{min}^{-1}$. 
A quasi-Binary Phase Diagram was constructed by plotting onset and endset of melting obtained from the DSC data performed on a set of elven samples (table SI-2, fig. SI-1). The samples were prepared by ball milling of different molar fractions of $R$-MAN $R$-ETI and $S$ MAN.S-ETI using the same milling conditions as for conglomerate identification (see 1.2).

Table SI-1. Data set used to build quasi-Binary melting Phase diagram for $R$-MAN $R$-ETI and S-MAN·S-ETI.

\begin{tabular}{cccc}
\hline Ratio $R$-MAN.R-ETI & Ratio S-MAN.S-ETI & $\mathbf{T}_{\text {onset }}\left({ }^{\circ} \mathbf{C}\right)$ & $\mathbf{T}_{\text {endset }}\left({ }^{\circ} \mathbf{C}\right)$ \\
\hline 1.0 & 0.0 & 93.09 & --- \\
0.9 & 0.1 & 53.45 & 89.68 \\
0.8 & 0.2 & 53.99 & 83.64 \\
0.7 & 0.3 & 54.74 & 76.70 \\
0.6 & 0.4 & 55.30 & 65.14 \\
0.5 & 0.5 & 55.44 & 60.64 \\
0.4 & 0.6 & 55.52 & 68.75 \\
0.3 & 0.7 & 55.84 & 75.24 \\
0.2 & 0.8 & 54.70 & 82.95 \\
0.1 & 0.9 & 53.53 & 89.27 \\
0.0 & 1.0 & 93.38 & --- \\
\hline
\end{tabular}

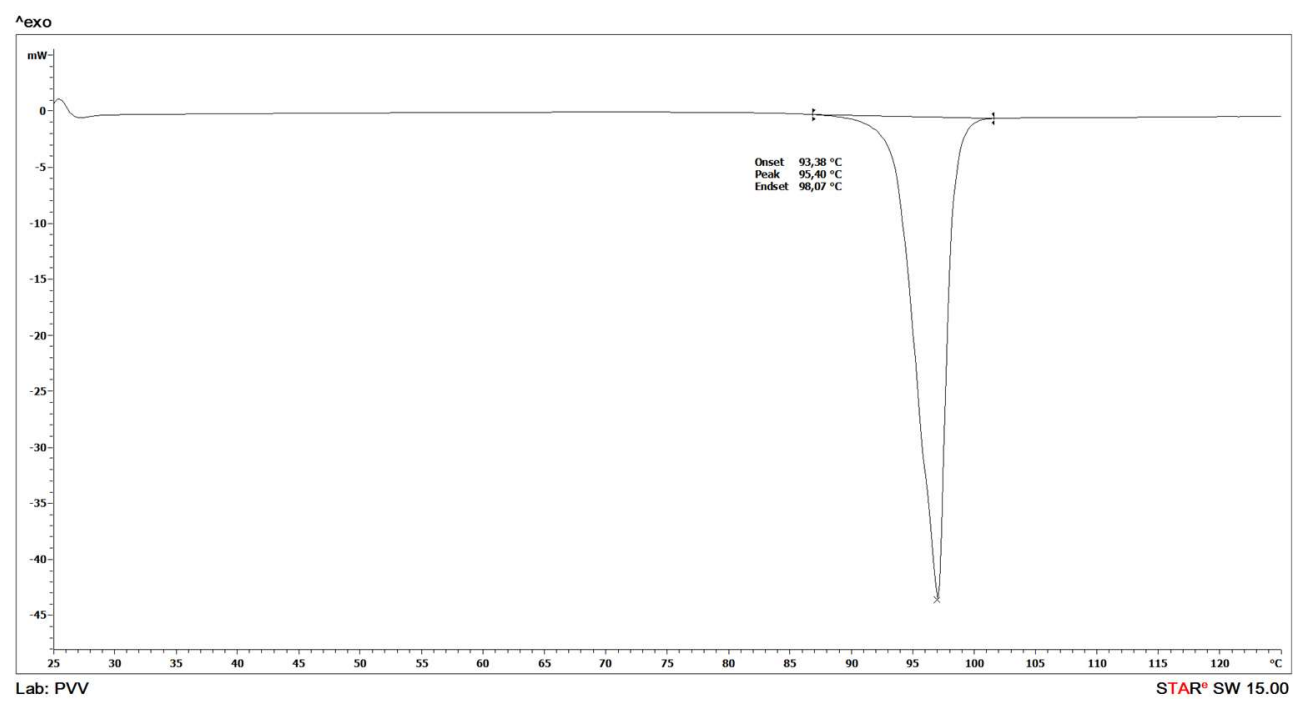

1) DSC thermogram of S-MAN.S-ETI. 


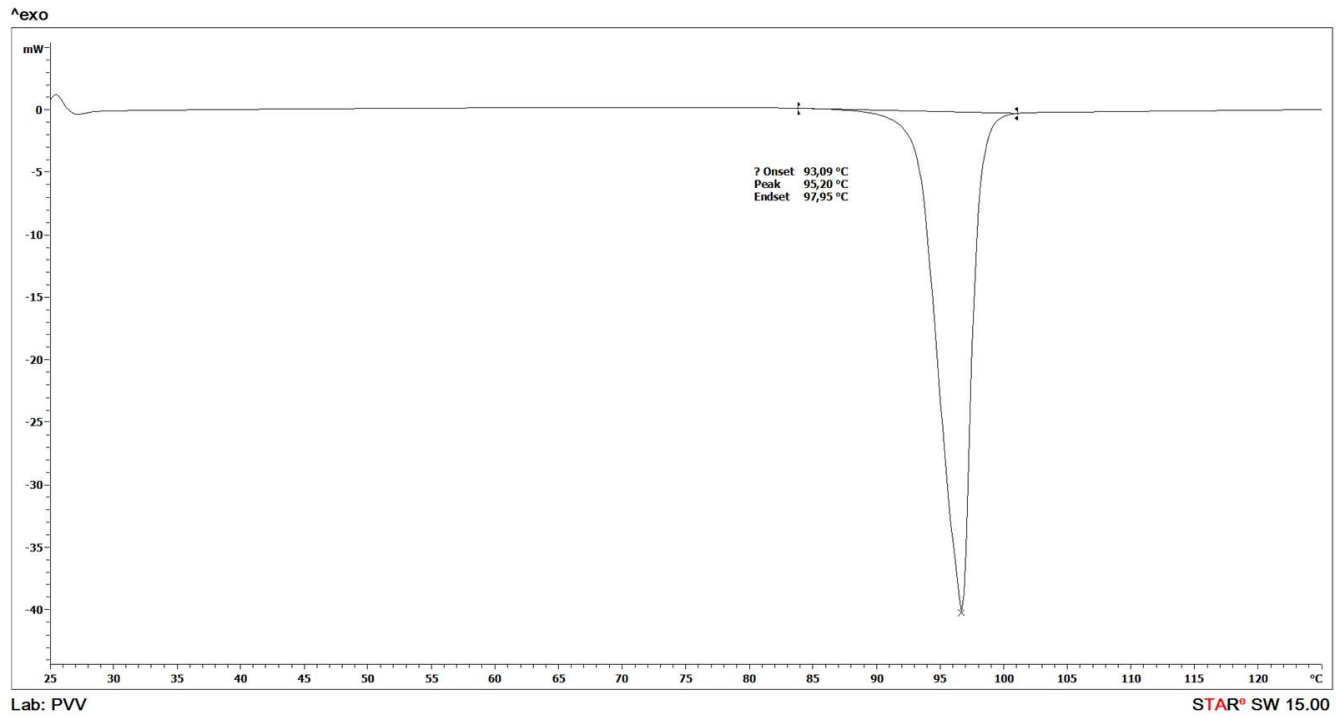

2) DSC thermogram of $R-\mathrm{MAN} \cdot R-\mathrm{ETI}$.

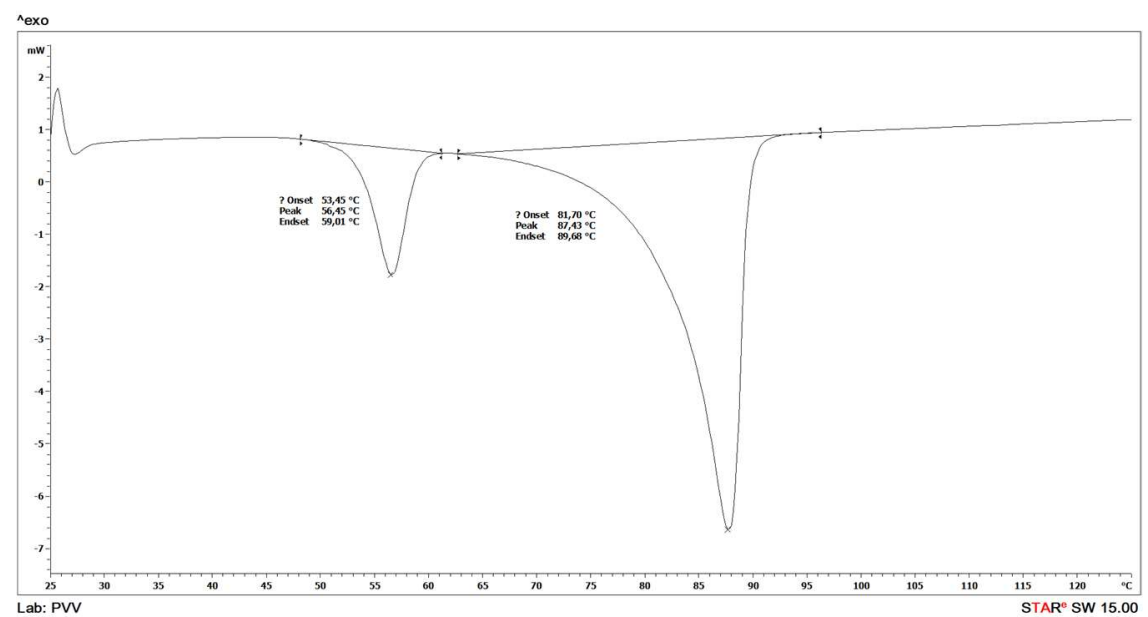

3) DSC thermogram of $R$-MAN.R-ETI / S-MAN.S-ETI in 9:1 ratio.

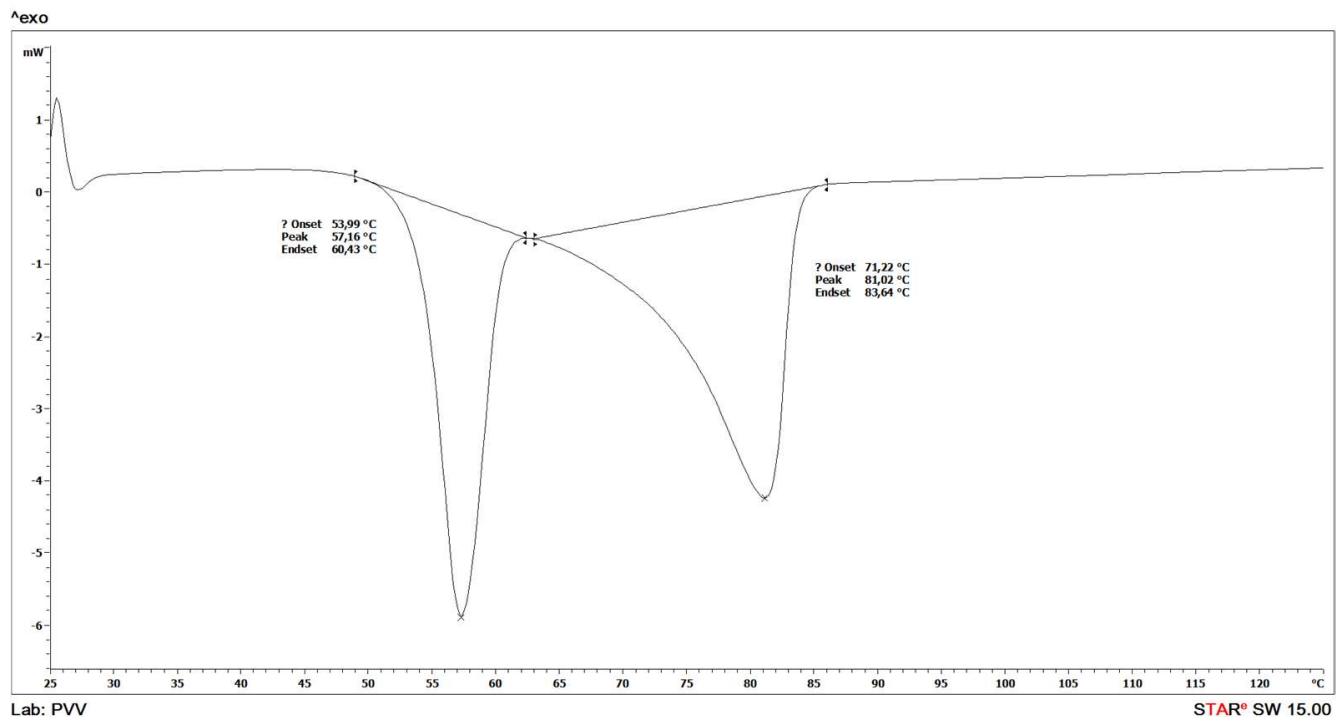

4) $\mathrm{DSC}$ thermogram of $R$-MAN $\cdot R$-ETI / S-MAN.S-ETI in 8:2 ratio. 


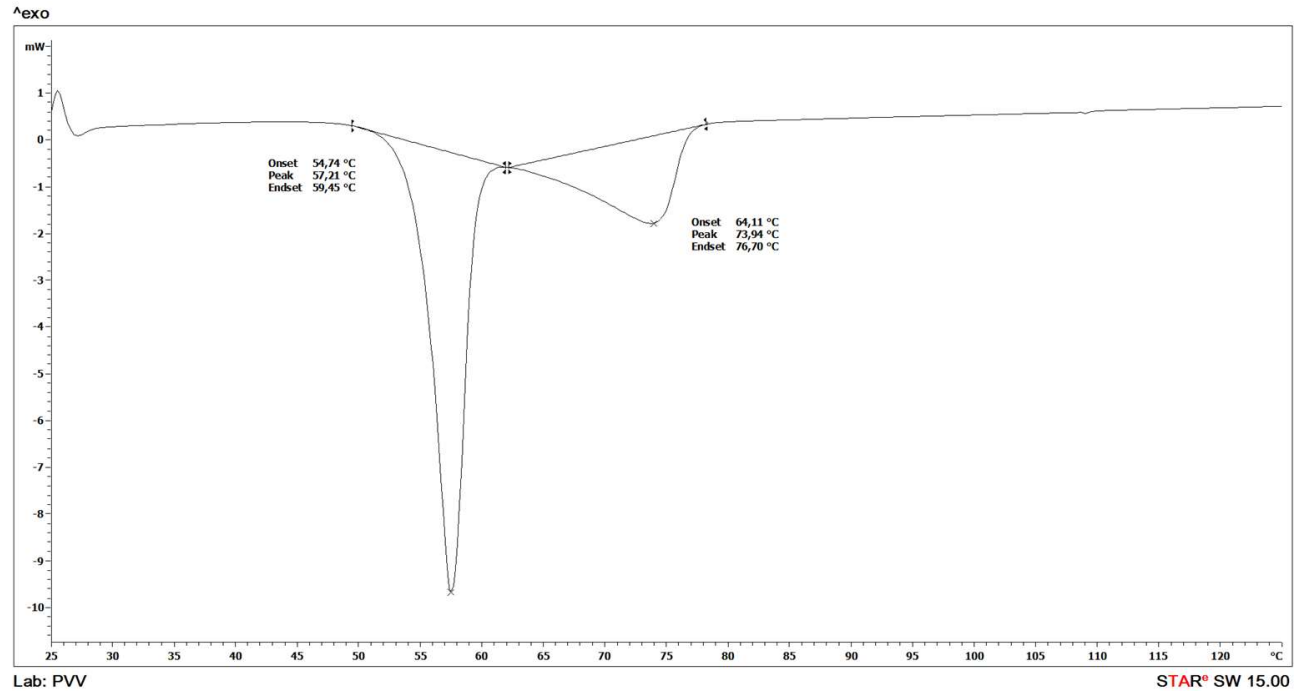

5) DSC thermogram of $R$-MAN.R-ETI / S-MAN.S-ETI in 7:3 ratio.

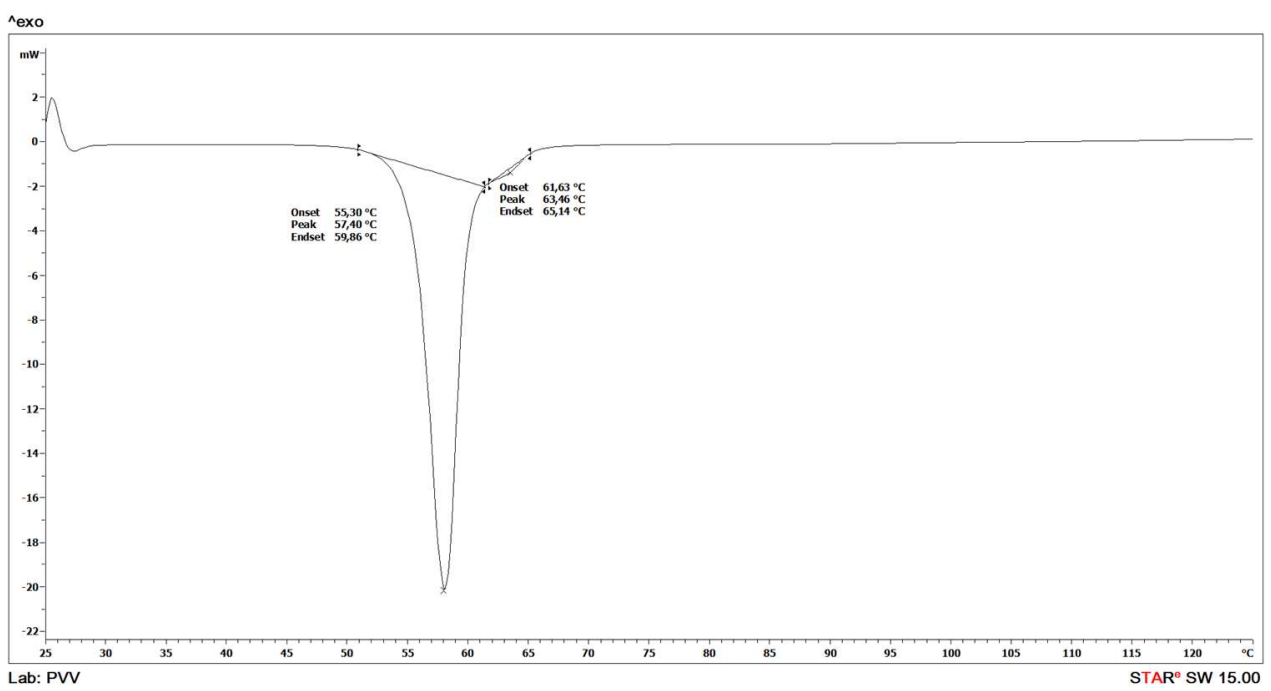

6) DSC thermogram of $R$-MAN $R$-ETI / S-MAN.S-ETI in 6:4 ratio.

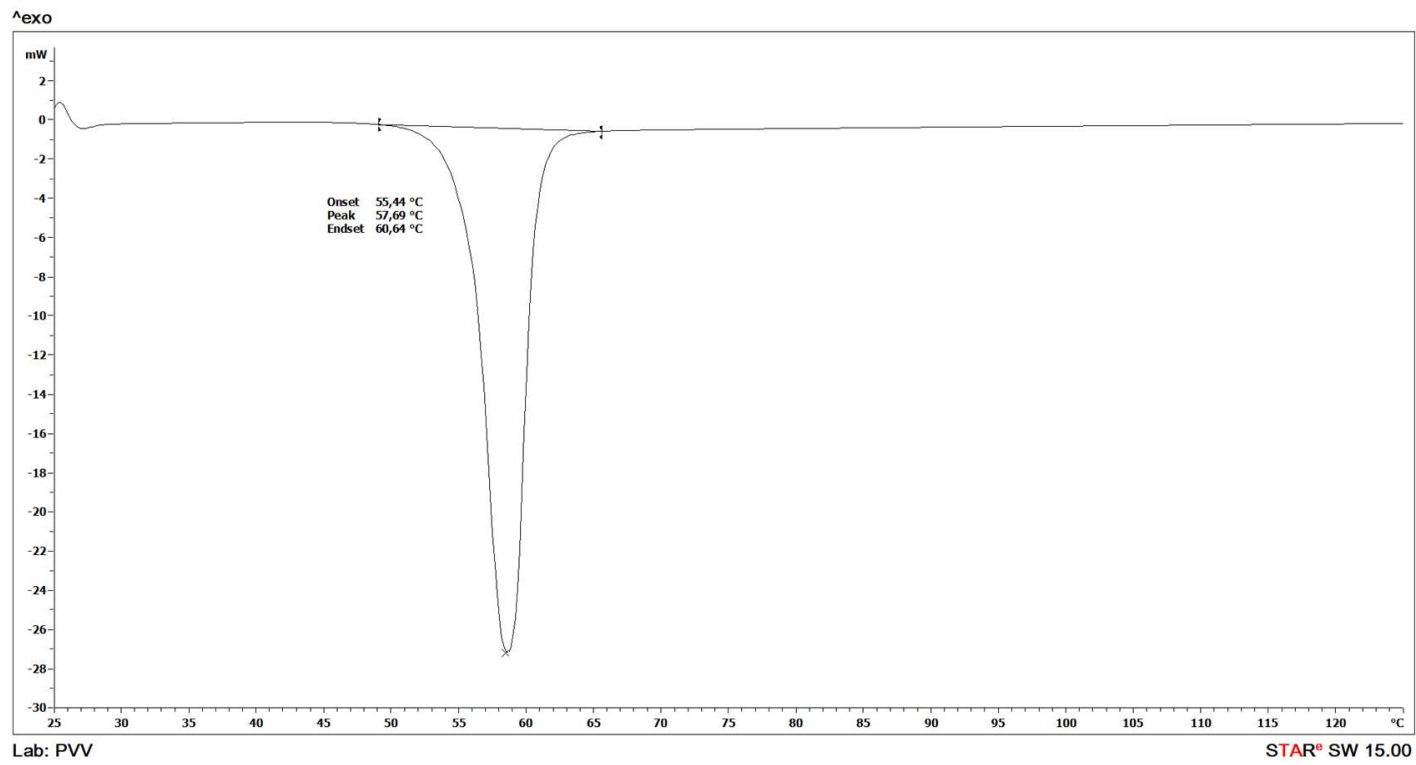

7) DSC thermogram of $R$-MAN.R-ETI / S-MAN.S-ETI in 1:1 ratio. 


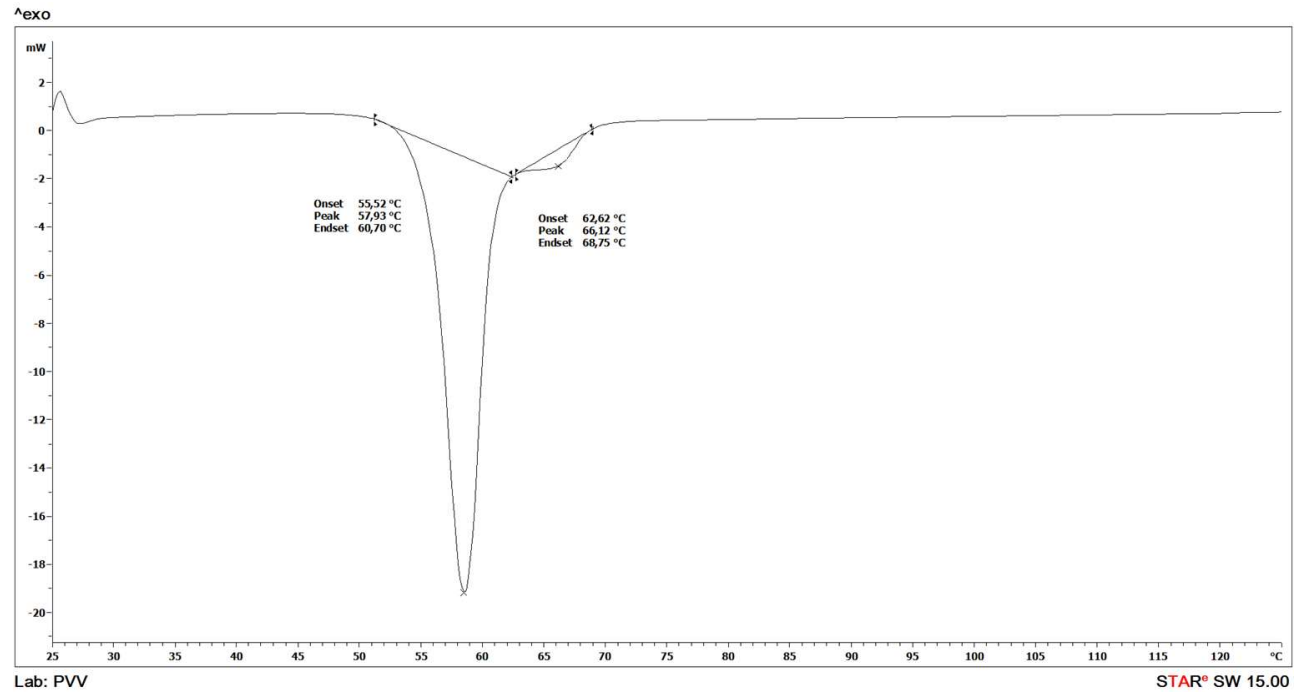

8) DSC thermogram of $R$-MAN.R-ETI / S-MAN.S-ETI in 4:6 ratio.

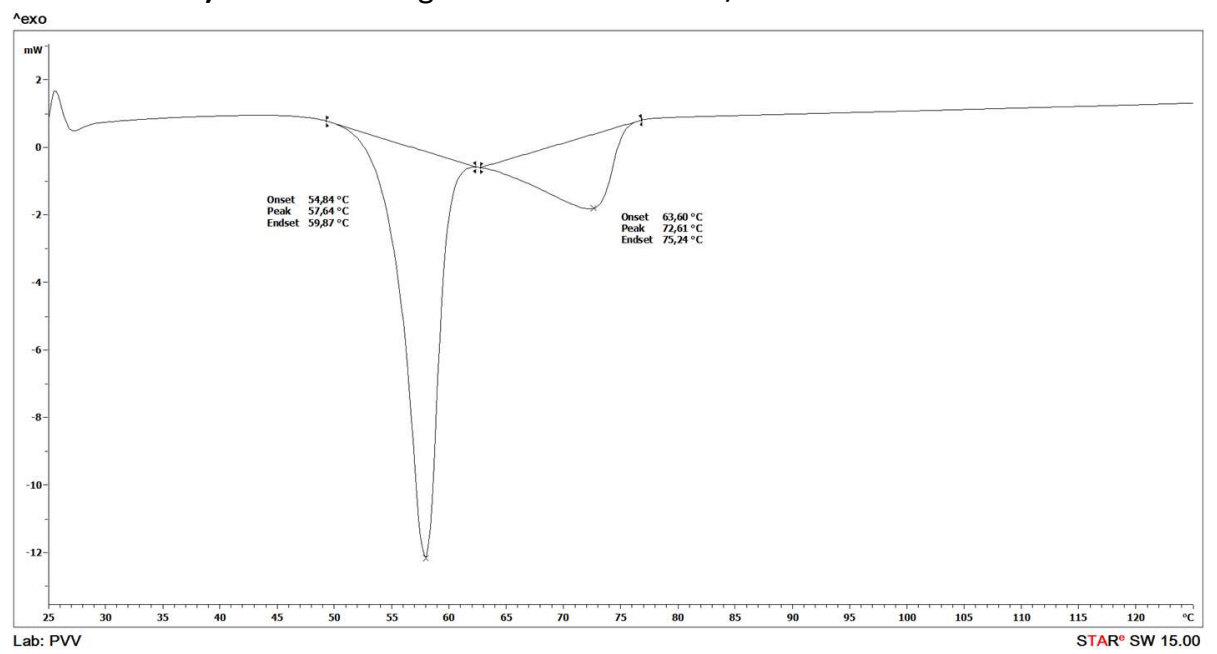

9) DSC thermogram of $R$-MAN $\cdot R$-ETI / S-MAN.S-ETI in 3:7 ratio.

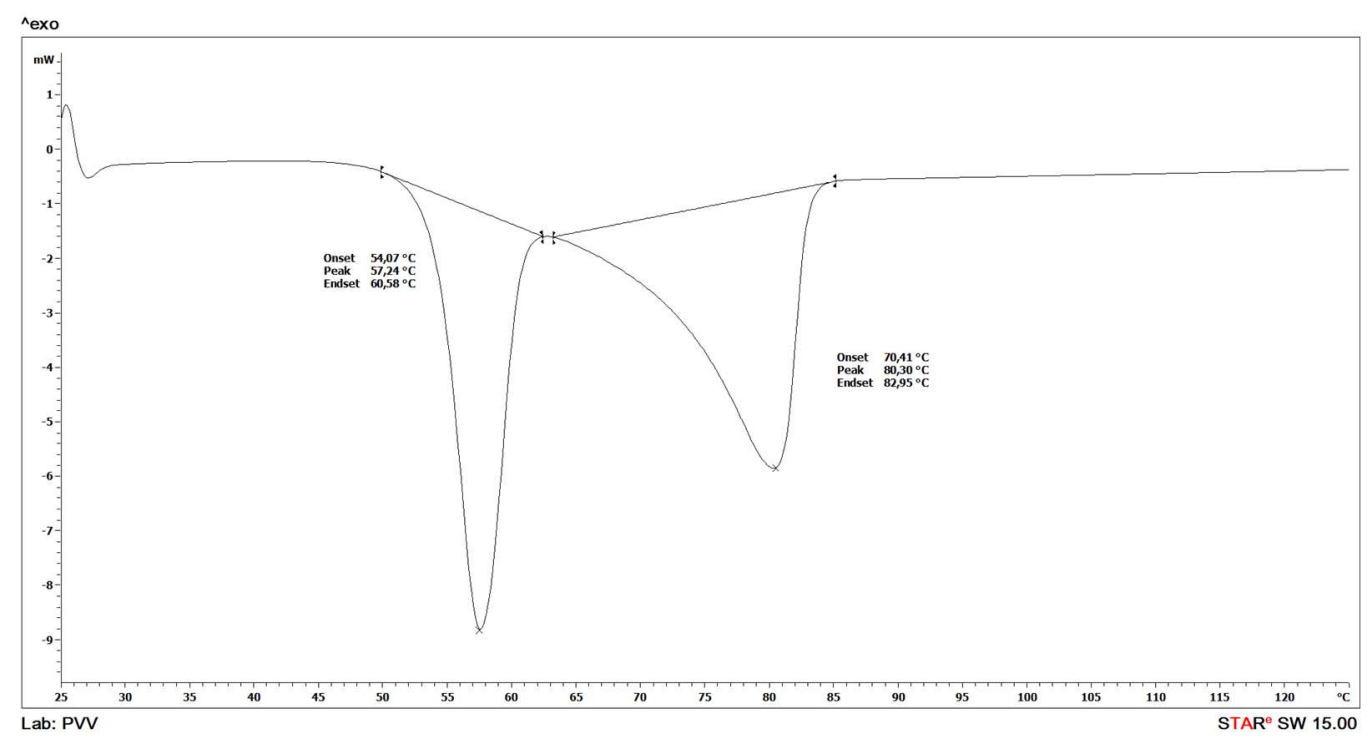

10) DSC thermogram of $R$-MAN.R-ETI / S-MAN.S-ETI in 2:8 ratio. 


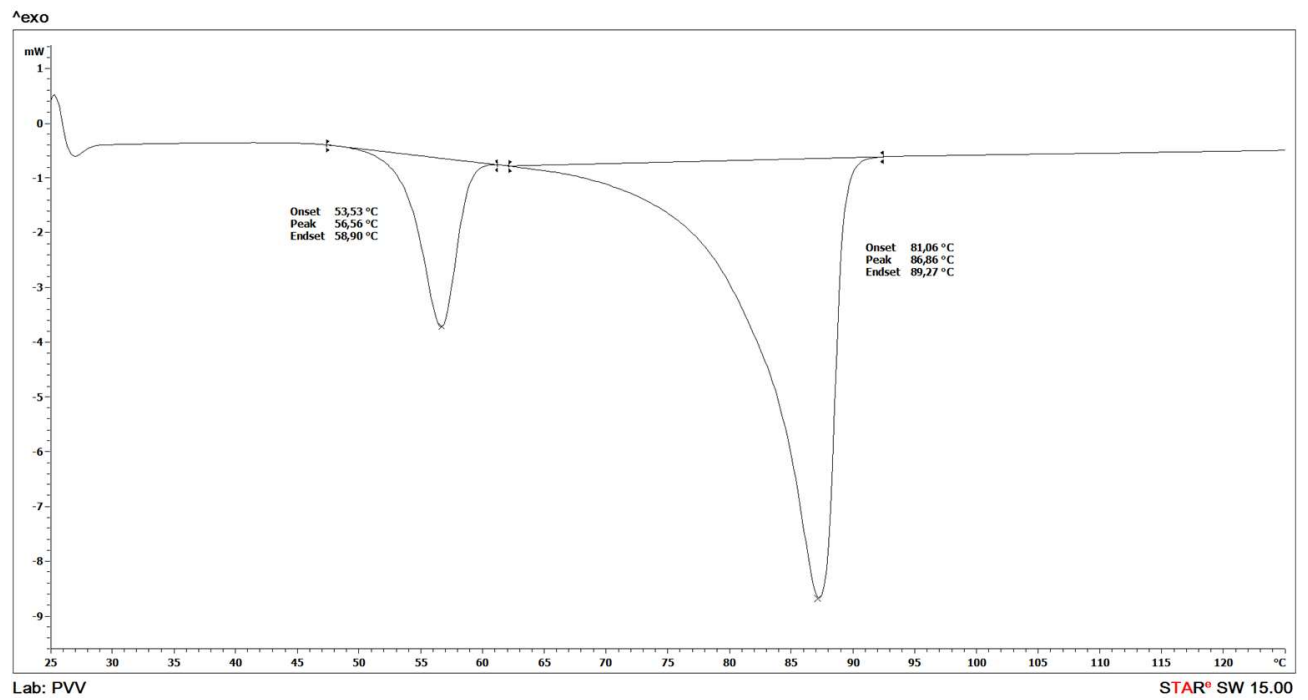

11) DSC thermogram of $R$-MAN $R$-ETI / S-MAN-S-ETI in $1: 9$ ratio.

Fig. SI-1. DSC thermograms used for the construction of the Binary Phase Diagram.

\section{Solubility curves}

Solubility Curves of S-MAN.S-ETI and of R-MAN-R-ETI / S-MAN·S-ETI were obtained weighing the solids in increasing amounts in vials of $1.5 \mathrm{~mL}$, topped with $1 \mathrm{~mL}$ of toluene and then sealed. Each vial was equipped with a stirring magnet. A heating-cooling cycle was then applied on the vials from 0 to $60{ }^{\circ} \mathrm{C}$ with a heating/cooling rate of $0.05^{\circ} \mathrm{C} \cdot \mathrm{min}^{-1}$ and under a continuous stirring of ca. 700rpm. Using a Crystal16 crystallization system (Technobis), turbidity was continuously monitored, allowing to identify clear point temperatures of the overall sample compositions. The average clear point temperatures over three measurements were assumed to be equal to the saturation temperature of these compositions. Solubility is expressed in $\mathrm{mg} \cdot \mathrm{mL}^{-1}$ (fig. SI-2, tables $\mathrm{SI}-3$ and SI-4).

Table SI-3. Solubility data for S-MAN·S-ETI.

\begin{tabular}{cc}
\hline $\mathbf{T},{ }^{\circ} \mathbf{C}$ & $\begin{array}{c}\text { Concentration } \\
\mathbf{m g} \cdot \mathbf{m L}^{-1}\end{array}$ \\
\hline 30.9 & 3.55 \\
33.1 & 3.95 \\
34.8 & 4.51 \\
37.0 & 5.12 \\
37.6 & 5.98 \\
40.5 & 6.85 \\
41.6 & 7.89 \\
43.6 & 9.71 \\
\hline
\end{tabular}


Table SI-4. Solubility data for R-MAN·R-ETI / S-MAN·S-ETI conglomerate in toluene.

\begin{tabular}{cc}
\hline $\mathbf{T}^{\circ}{ }^{\circ} \mathbf{C}$ & $\begin{array}{c}\text { Concentration, } \\
\mathrm{mg} \cdot \mathrm{mL}^{-1}\end{array}$ \\
\hline 18.1 & 8.67 \\
23.7 & 14.48 \\
28.4 & 26.53 \\
29.0 & 28.70 \\
29.6 & 31.39 \\
30.0 & 35.03 \\
30.5 & 39.02 \\
31.2 & 43.87 \\
31.9 & 50.72 \\
32.1 & 56.36 \\
32.9 & 73.70 \\
33.5 & 86.70 \\
34.1 & 108.38 \\
\hline
\end{tabular}

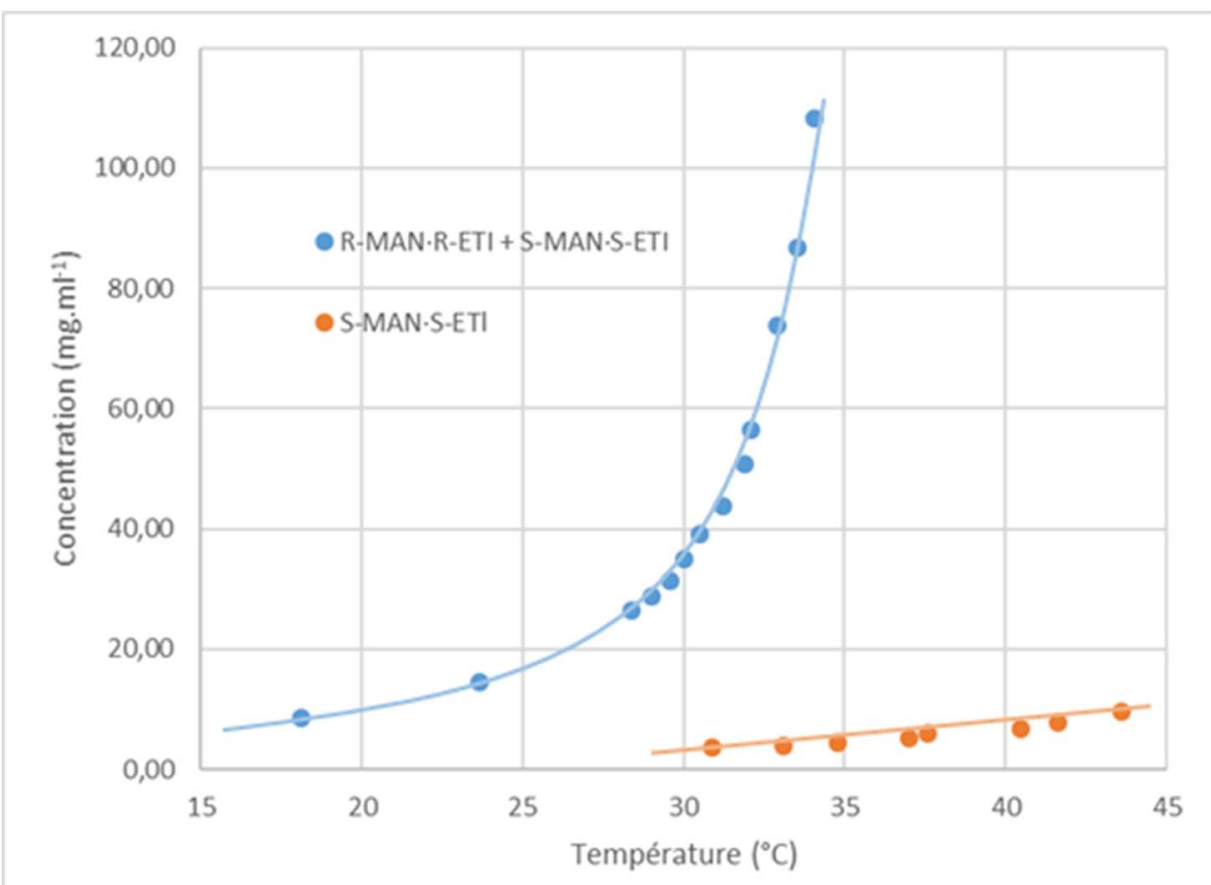

Fig. SI-2. Solubility curves of equimolar composition of conglomerate S-MAN-S-ETI/R-MAN-R-ETI (red) and enantiopure S-MAN-S-ETI cocrystal (blue) in toluene.

The cocrystal system presented here shows a significant deviation from Meyerhoffer's doublesolubility rule, ${ }^{[3]}$ with the conglomerate $>10$ times more soluble at $30^{\circ} \mathrm{C}$ with respect to the enantiopure cocrystal. This is likely due to a very strong intermolecular interaction in solution between coformers of opposite chirality (e. g. S-MAN and R-ETI), explaining a strong increase in solubility for the conglomerate. 


\section{Quasi-Ternary isothermal phase diagram}

The quasi-ternary isothermal phase diagram was constructed by slurrying $R$-MAN $R$-ETI and S-MAN.S-ETI in toluene in Hettich Benelux Cooling Thermo Shaker MKR 13, varying the molar ratio of the corresponding enantiopure cocrystals from 0 to 1 (fig. SI-3). Experiments were carried out in $5 \mathrm{~mL}$ sealed vials, and the suspensions were stirred at $25^{\circ} \mathrm{C}$ over $48 \mathrm{~h}$. Seeding with all possible solid-state forms was performed to make sure that the system reached thermodynamic equilibrium. Afterwards the samples were filtered by sand core funnel. The solid phases were characterized by XRPD.

To determine the solubility line, each experimental condition described above was repeated, but this time the samples were not filtered; small portions of toluene were added to the stirring mixtures every 2 hours, until complete dissolution of the suspended solid was observed, at which point the liquidus line was reached. The TPD has been drawn using ProSim Ternary Diagram software.

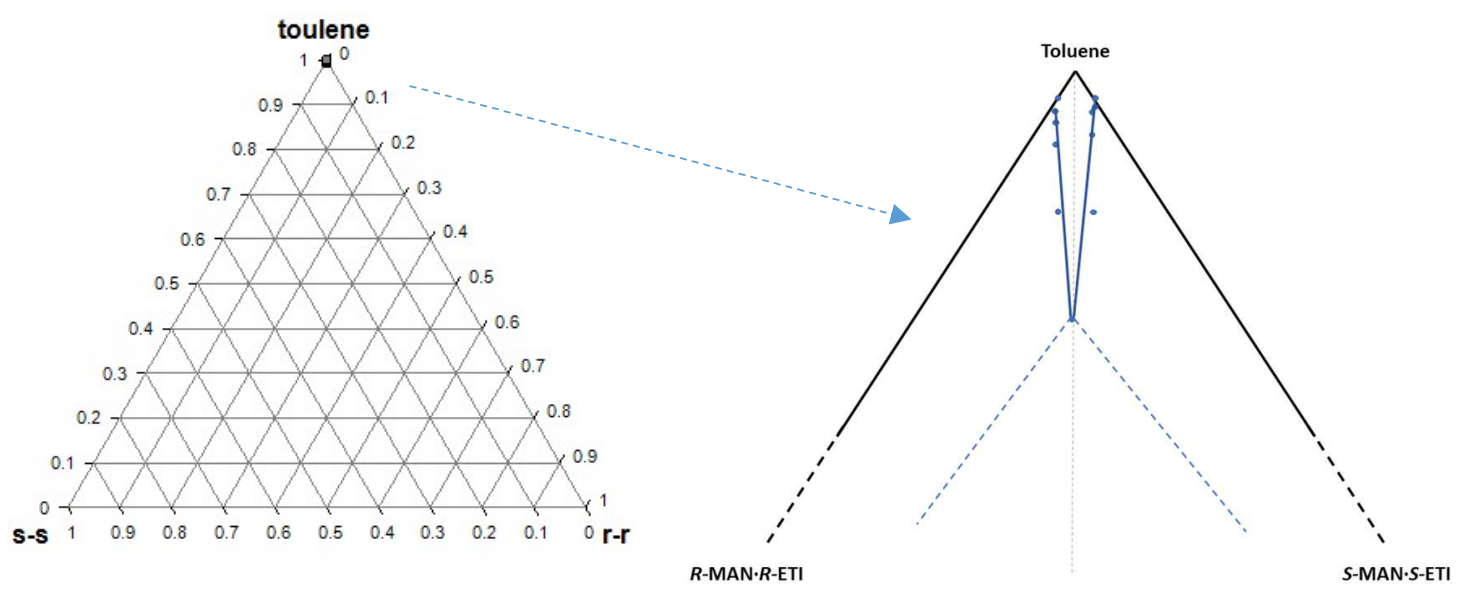

Fig. SI-3. Quasi-Ternary isothermal phase diagram of S-MAN-S-ETI, R-MAN-R-ETI and toluene

Table SI-5. Experimental mass fractions (S-MAN-S-ETI, R-MAN·R-ETI and toluene) used to build the Ternary Phase Diagram.

\begin{tabular}{|c|c|c|c|c|c|c|c|c|}
\hline \multirow{2}{*}{$\begin{array}{c}\text { Initial } \\
\text { composition } \\
R \text {-MAN·R-ETI: } \\
S \text {-MAN-S-ETI } \\
(\%)\end{array}$} & \multirow{2}{*}{$\begin{array}{c}\text { Toluene } \\
\text { initial } \\
\text { (g) }\end{array}$} & \multirow{2}{*}{$\begin{array}{c}\text { Toluene } \\
\text { added } \\
\text { (g) }\end{array}$} & \multicolumn{3}{|c|}{$\begin{array}{l}\text { Composition at dissolution point } \\
\text { (g) }\end{array}$} & \multicolumn{3}{|c|}{$\begin{array}{l}\text { Composition at dissolution point } \\
\text { (mol \%) }\end{array}$} \\
\hline & & & $\begin{array}{c}R \text {-MAN. } \\
R \text {-ETI } \\
\left(* 10^{-3}\right)\end{array}$ & $\begin{array}{c}\text { S-MAN. } \\
S \text {-ETI } \\
\left(* 10^{-3}\right)\end{array}$ & Toluene & $\begin{array}{c}R \text {-MAN. } \\
R \text {-ETI } \\
\left({ }^{*} 10^{-4}\right)\end{array}$ & $\begin{array}{c}\text { S-MAN. } \\
\text { S-ETI } \\
\left(* 10^{-4}\right)\end{array}$ & Toluene \\
\hline $0: 100$ & 0.5 & 0.705 & 2.7 & 0 & 1.205 & 0 & 0.637 & 0.9993 \\
\hline 10:90 & 0.5 & 3.1 & 1 & 9 & 3.6 & 0.710 & 0.789 & 0.9992 \\
\hline $20: 80$ & 0.5 & 2.73 & 2 & 8 & 3.23 & 0.704 & 0.176 & 0.9991 \\
\hline
\end{tabular}




\begin{tabular}{|l|l|c|c|c|c|c|c|c|}
\hline $\mathbf{3 0 : 7 0}$ & 0.5 & 1.61 & 3 & 7 & 2.11 & 0.942 & 0.404 & 0.9987 \\
\hline $\mathbf{4 0 : 6 0}$ & 0.5 & 0.45 & 4 & 6 & 0.95 & 1.79 & 1.19 & 0.9970 \\
\hline $\mathbf{5 0 : 5 0}$ & 0.5 & 1.29 & 16.1 & 16.1 & 1.79 & 2.54 & 2.54 & 0.9949 \\
\hline $\mathbf{6 0 : 4 0}$ & 0.5 & 0.456 & 6 & 4 & 0.956 & 1.19 & 1.78 & 0.9970 \\
\hline $\mathbf{7 0 : 3 0}$ & 0.5 & 1.361 & 7 & 3 & 1.861 & 4.58 & 1.07 & 0.9984 \\
\hline $\mathbf{8 0 : 2 0}$ & 0.5 & 2.1 & 8 & 2 & 2.6 & 0.219 & 0.874 & 0.9989 \\
\hline $\mathbf{9 0 : 1 0}$ & 0.5 & 2.929 & 9 & 1 & 3.429 & 0.829 & 0.746 & 0.9991 \\
\hline $\mathbf{1 0 0 : 0}$ & 0.5 & 0.615 & 0 & 2.4 & 1.115 & 0.612 & 0 & 0.9993 \\
\hline
\end{tabular}

\section{Chiral High Performance Liquid Chromatography (cHPLC)}

The separation of $R$-/S-mandelic acid and $R$-/S-etiracetam was performed by Chiral HPLC using a Waters Alliance 2695 coupled with a PDA996 detector.

Due to structural differences between mandelic acid and etiracetam, two distinct chromatographic methods were used to achieve the separation of respective enantiomers.

1) The resolution of etiracetam enantiomers was achieved using a Daicel Chiralpak IA column $4.6 \times 250 \mathrm{~mm} 5 \mu \mathrm{m}$ particle size with a mobile phase consisting of $80 \% \mathrm{v} / \mathrm{v}$ i-hexane and $20 \% \mathrm{v} / \mathrm{v}$ ethanol.

2) The mandelic acid enantiomers were separated on a Daicel Chiralpak IC column $4.6 \times 250 \mathrm{~mm}$ $5 \mu \mathrm{m}$ with a mix of $95 \% \mathrm{v} / \mathrm{v}$ i-hexane, $5 \% \mathrm{v} / \mathrm{v}$ Isopropanol and $0,1 \%$ of TFA as mobile phase. The flow rate used in both methods is $1 \mathrm{~mL} / \mathrm{min}$ at $25^{\circ} \mathrm{C}$.

\section{Preferential Crystallization Process}

The preferential crystallization experiments were performed using an "EasyMax 102 Advanced Workstation (Mettler Toledo)", equipped with two jacket flasks of $100 \mathrm{~mL}$, magnetic stirring and temperature control. The saturated solution for the seeded isothermal preferential crystallization (SIPC) mode was created by slurrying $R$-MAN $R$-ETI / S-MAN-S-ETI conglomerate suspension at a temperature of $30^{\circ} \mathrm{C}$. After the equilibrium was reached $(24 \mathrm{~h})$, the suspension was filtered, the filtrate cooled down to the crystallization temperature of $27^{\circ} \mathrm{C}$ and the homochiral seeds of either R-MAN.R-ETI or S-MAN-S-ETI were added to the crystallizer. The batch crystallization process was stopped after $30 \mathrm{~min}$. The solid phase was recovered through filtration, washed with cold toluene and analyzed using XRPD and HPLC. 


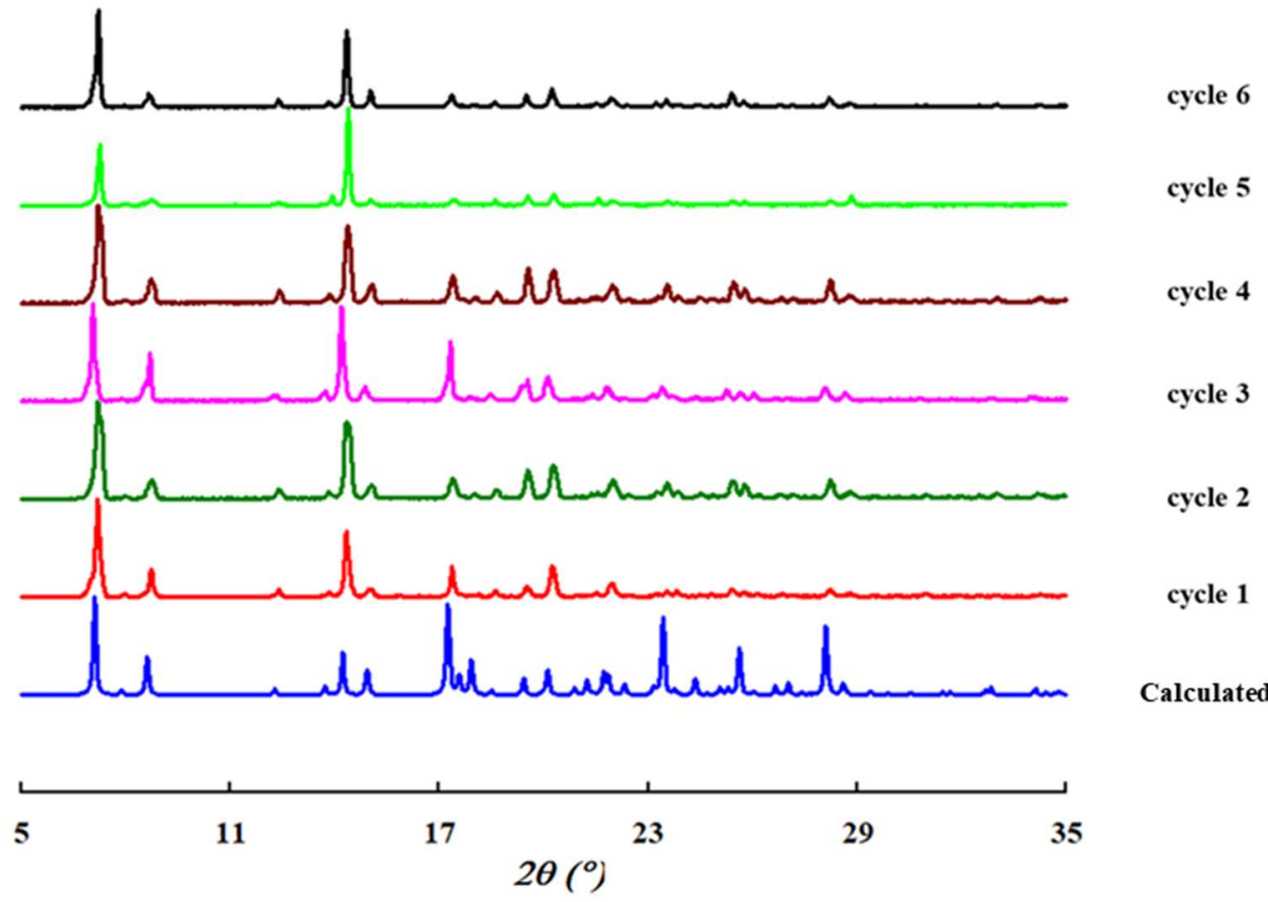

Fig. SI-4. Comparison of the experimental XRPD pattern of the solids obtained during the cyclic preferential crystallization experiment compared to the calculated pattern of S-MAN.S-ETI (CSD refcode YASGIK ${ }^{[2]}$ ).

1)
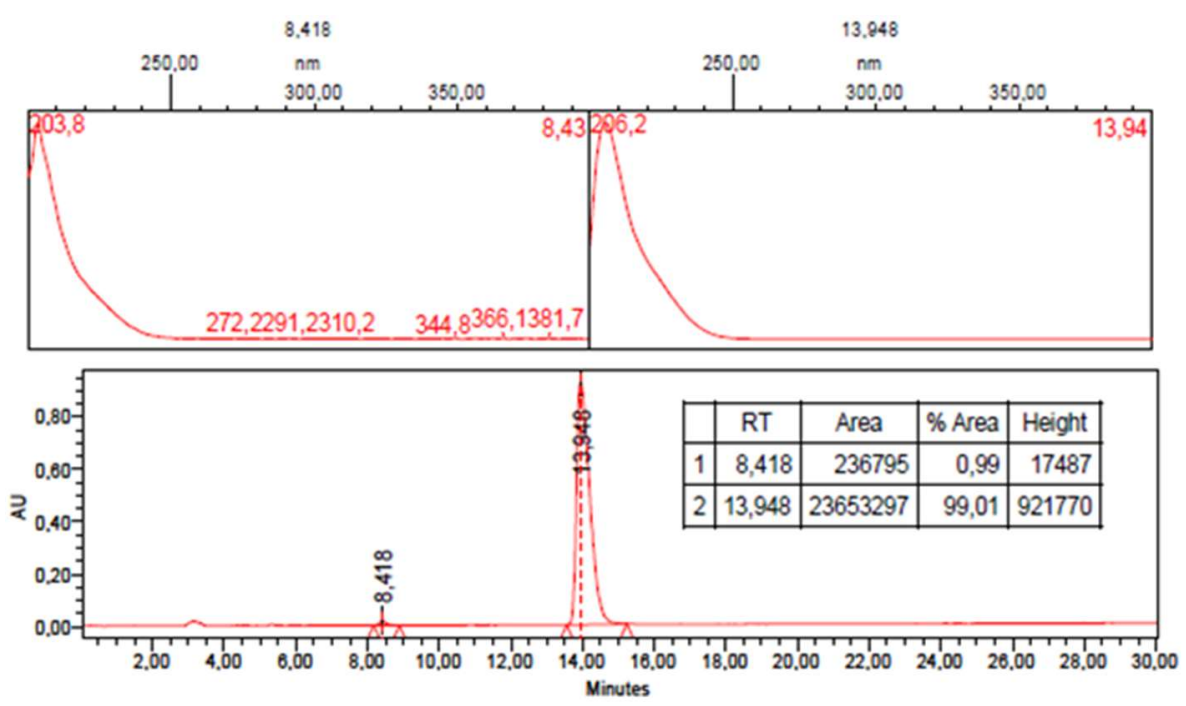
2)
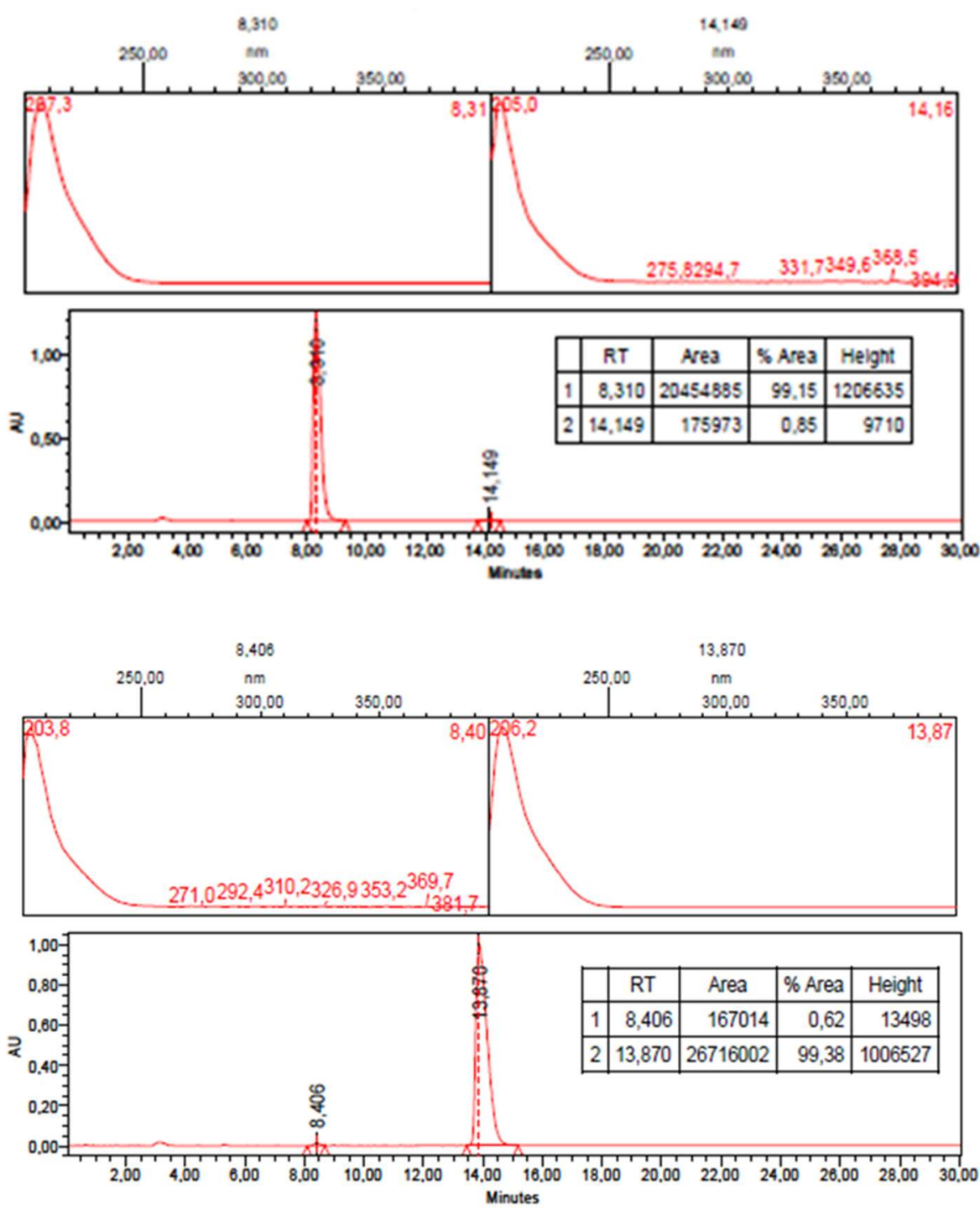

3)

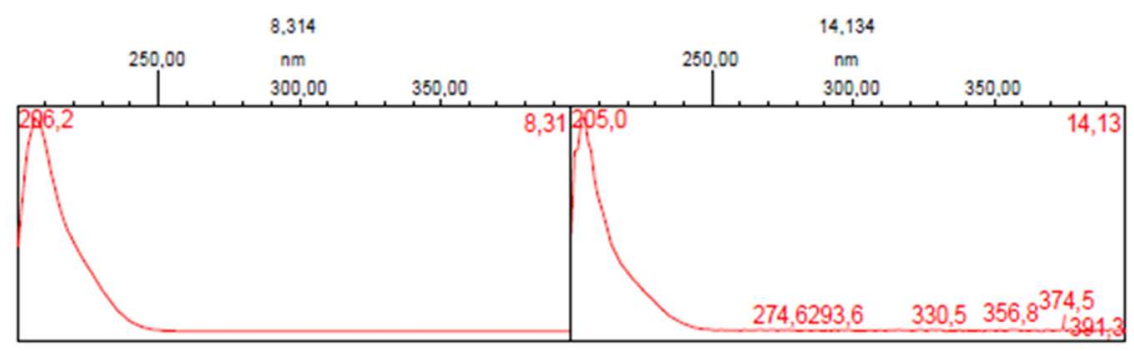

4)

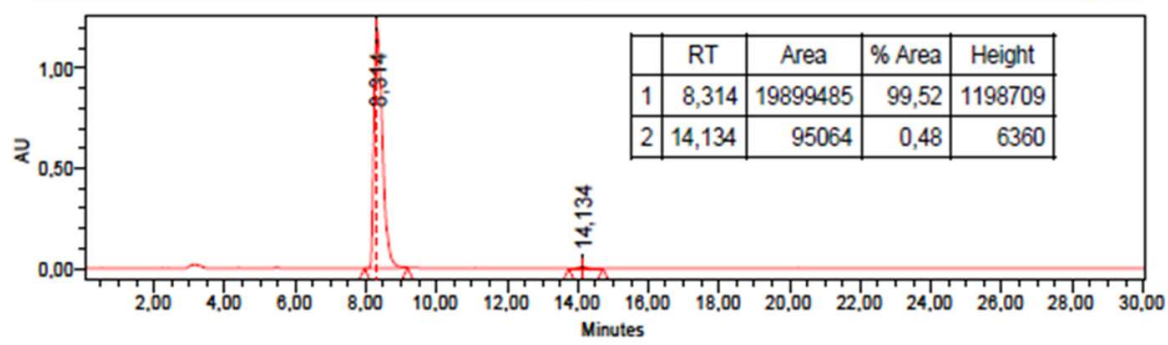



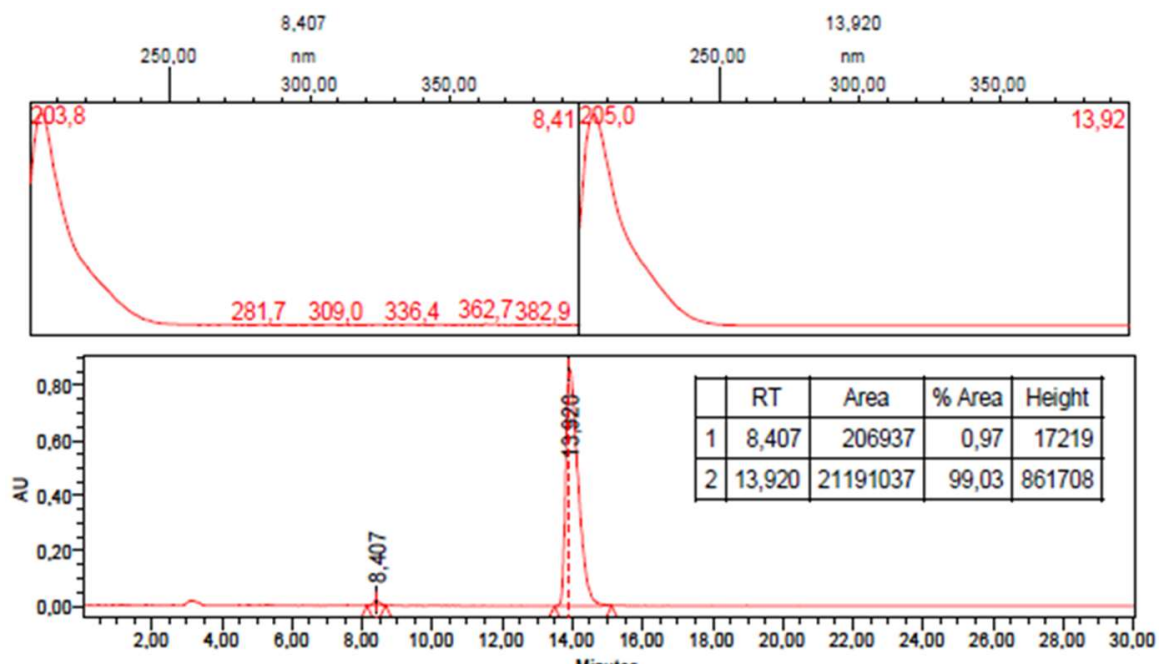

5) Minutes

6)
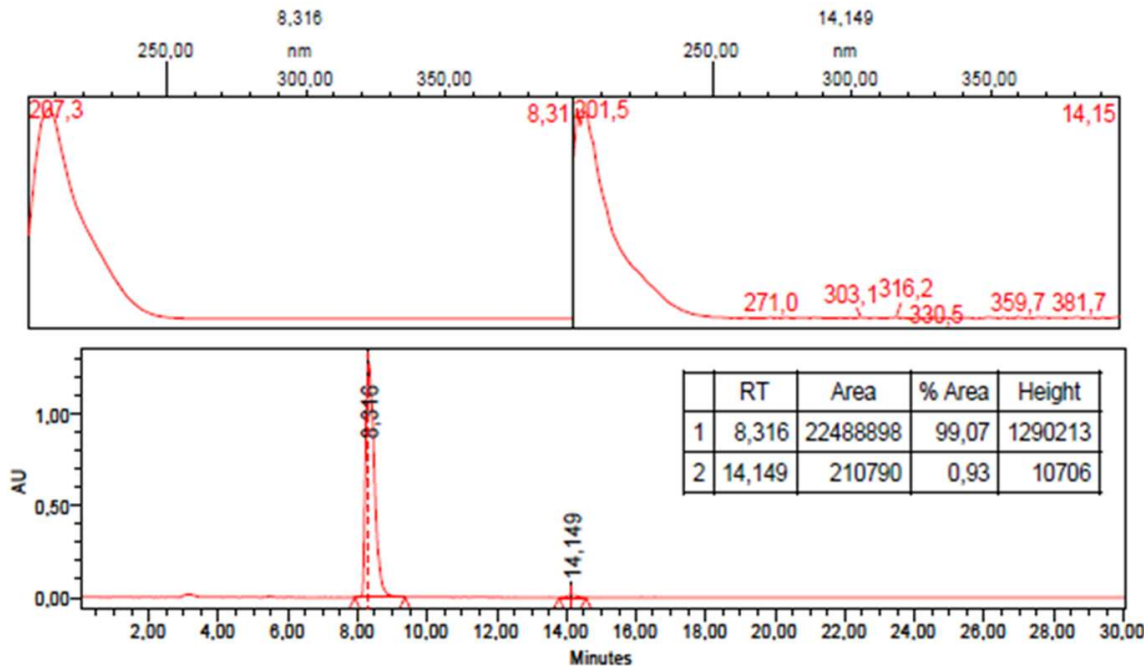

Fig. SI-5. UV spectrum index plot and reverse HPLC chromatograms used to evaluate the R-ETI/S-ETI ratio in Sduring the cyclic experiment (steps 1-6).

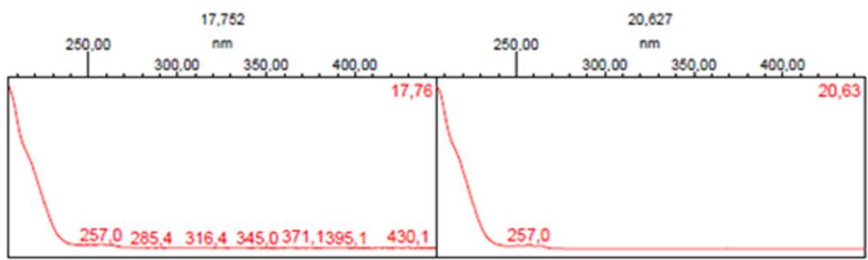

1)

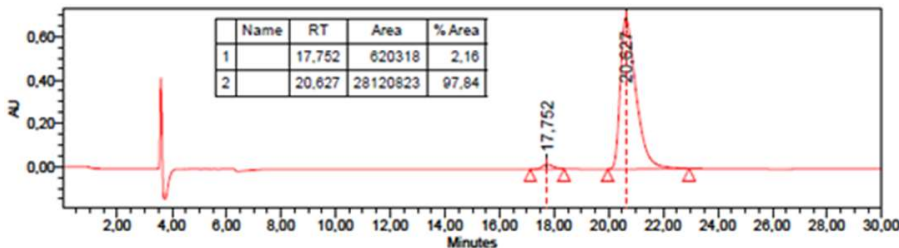




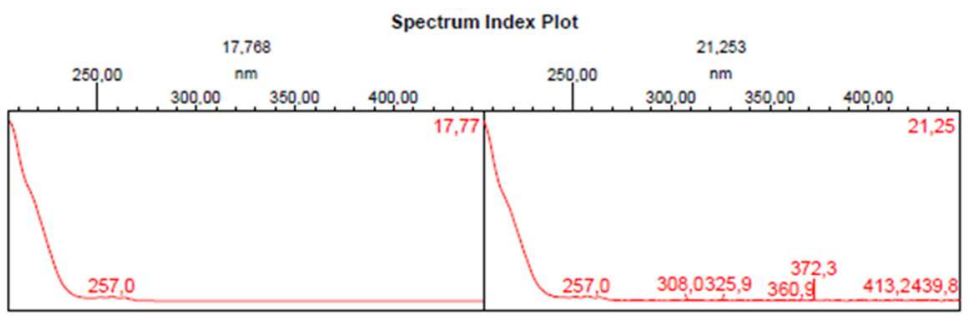

2)
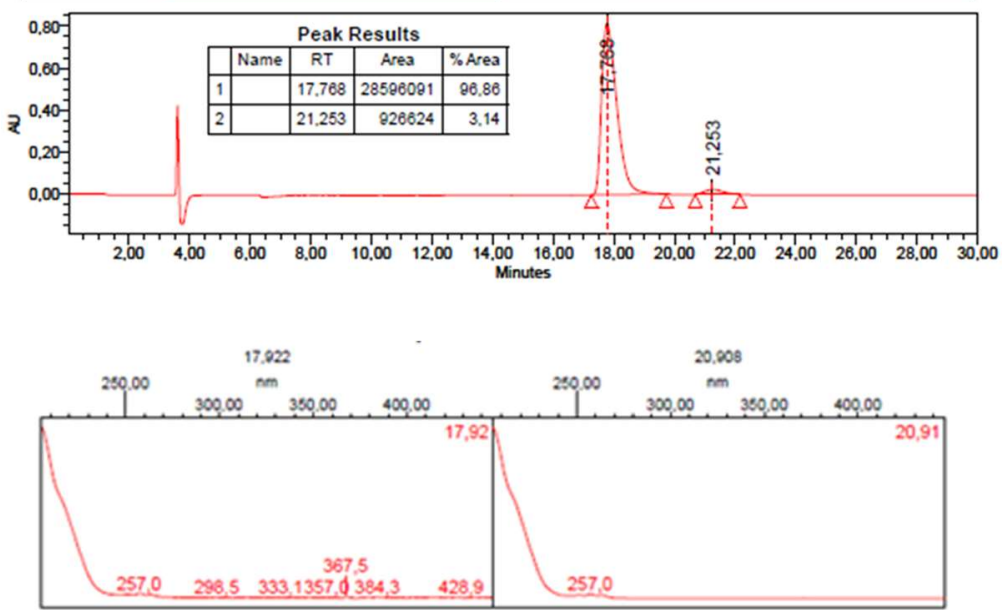

3)
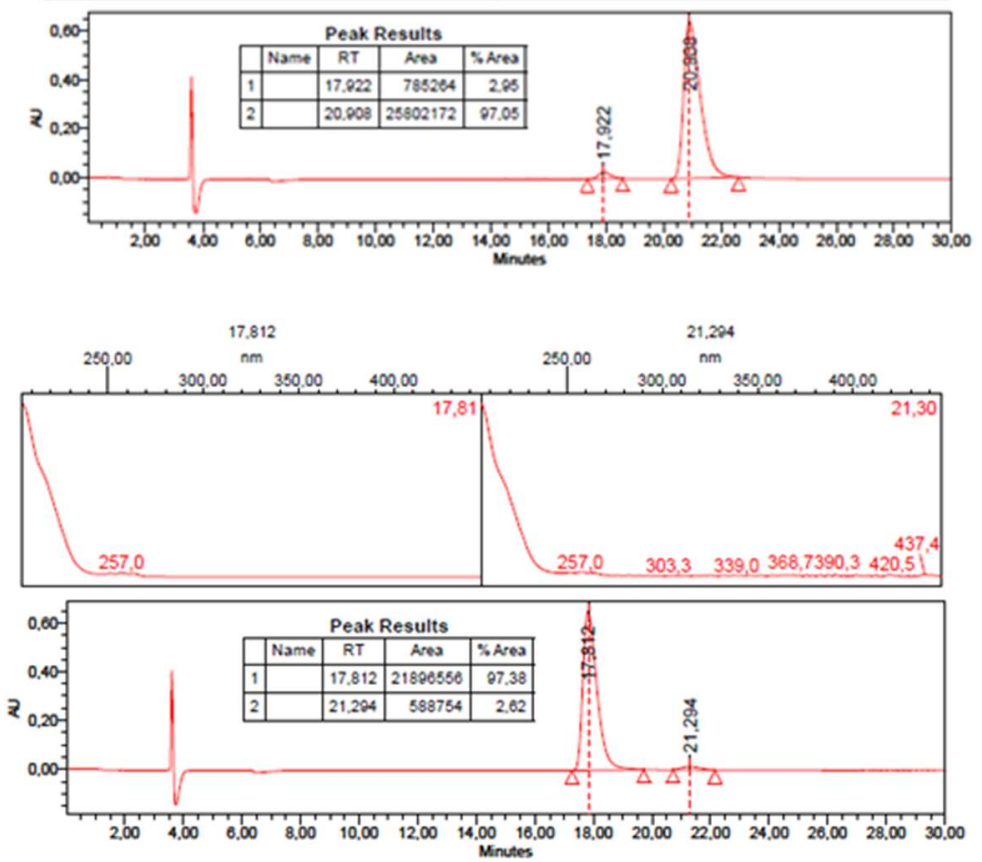

4)

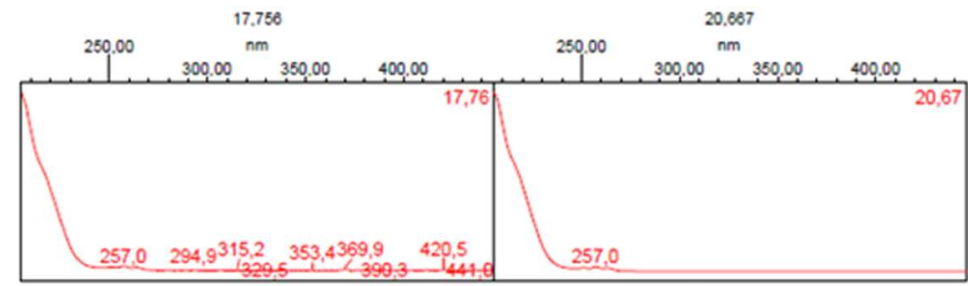

5)

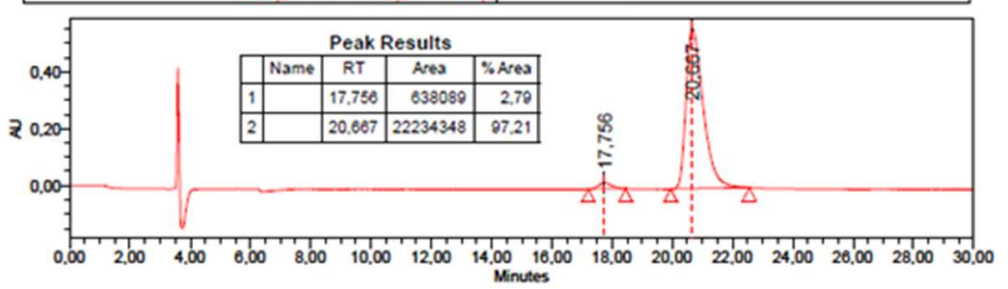


6)
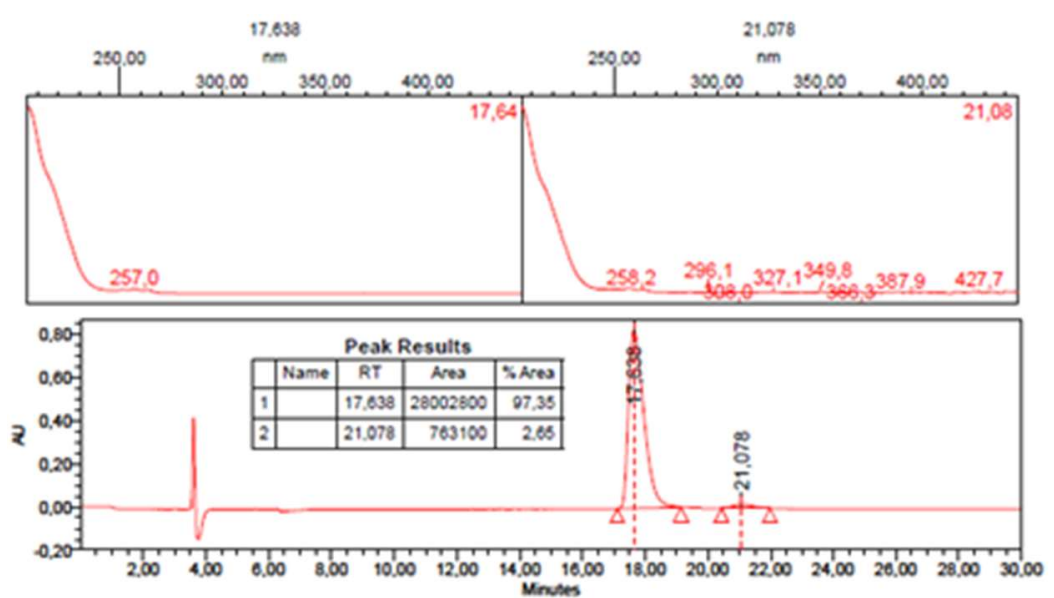

Fig. SI-6. UV spectrum index plot and reverse HPLC chromatograms used to evaluate the R-MAN/S-MAN ratio in the cyclic experiments (steps 1-6).

\section{Liquid and Solid Phase kinetic Follow-up}

For the kinetic follow-up of the process the SIPC was upscaled using a 1L-Reactor-Ready ${ }^{\mathrm{TM}}$ Lab Reactor from Radleys. $10 \mathrm{~mL}$ of the suspension was collected every 5-10 min and filtered. The filtrate thus obtained was left to evaporate. The initial solid in suspension as well as the solid obtained after evaporation of the filtrate, were analyzed by cHPLC to follow the ee over time (table SI-6-7 and fig. SI-7 and SI-8 correspondingly).

\section{Solid Phase kinetic Follow-up:}

Table SI-6. Solid Phase kinetic Follow-up

\begin{tabular}{cccc}
\hline Time after seed addition & Purity $\mathbf{R}_{\text {-ETI }}$ & Purity $\mathbf{R}_{\text {-ETI }}$ & ee of $\boldsymbol{R}$-MAN-R-ETI \\
\hline 0 & 100 & 0 & 100 \\
10 & 99.56 & 0.44 & 99.12 \\
20 & 99.57 & 0.43 & 99.14 \\
35 & 99.37 & 0.63 & 98.74 \\
45 & 90.45 & 9.55 & 80.9 \\
55 & 77.42 & 22.58 & 54.84 \\
65 & 61.62 & 38.38 & 23.24 \\
75 & 56.26 & 43.74 & 12.52 \\
85 & 57.04 & 42.92 & 14.12 \\
95 & 56.04 & 43.96 & 12.08 \\
105 & 54.52 & 45.48 & 9.04 \\
115 & 51.91 & 48.09 & 3.82 \\
155 & 51.79 & 48.21 & 3.58 \\
\hline
\end{tabular}




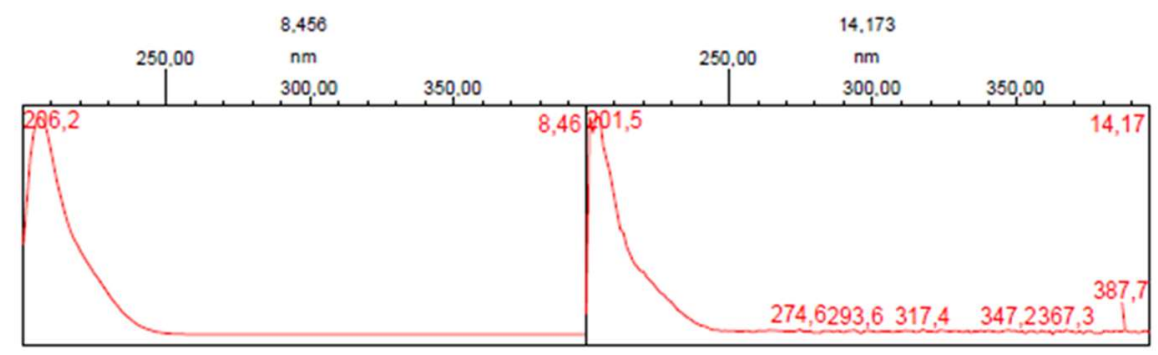

1) Solid : t=20 $\mathrm{min}$.
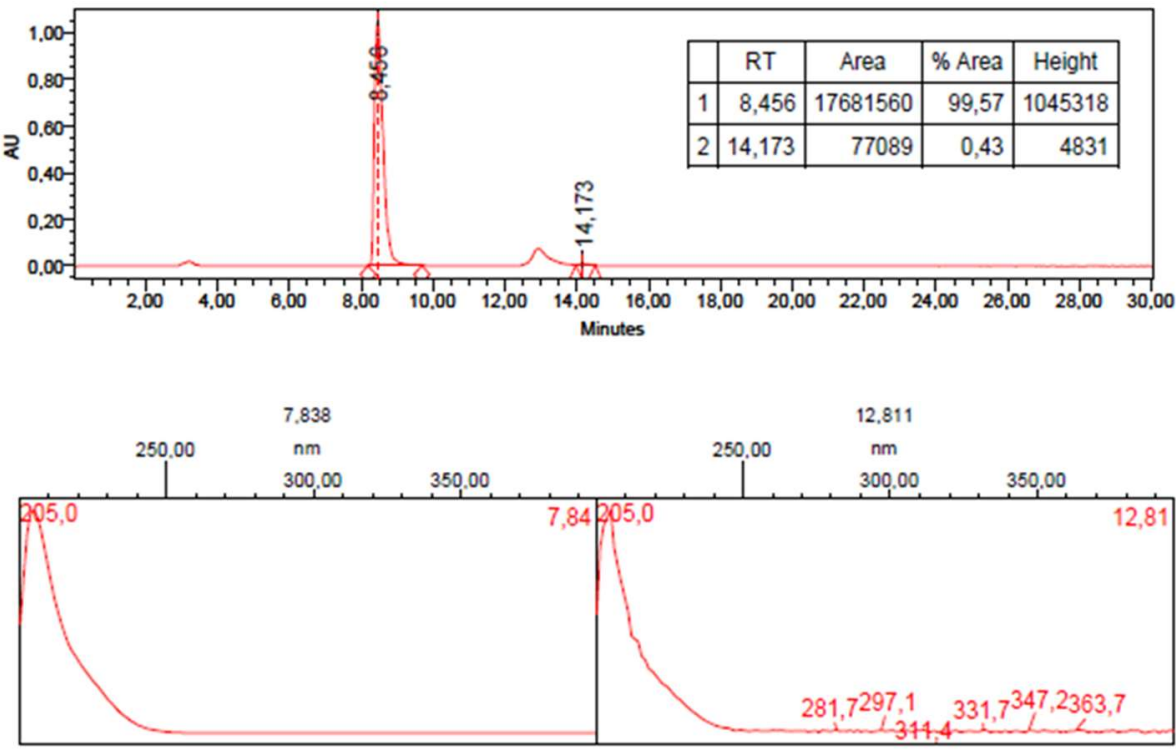

2) Solid : t=35 $\mathrm{min}$.

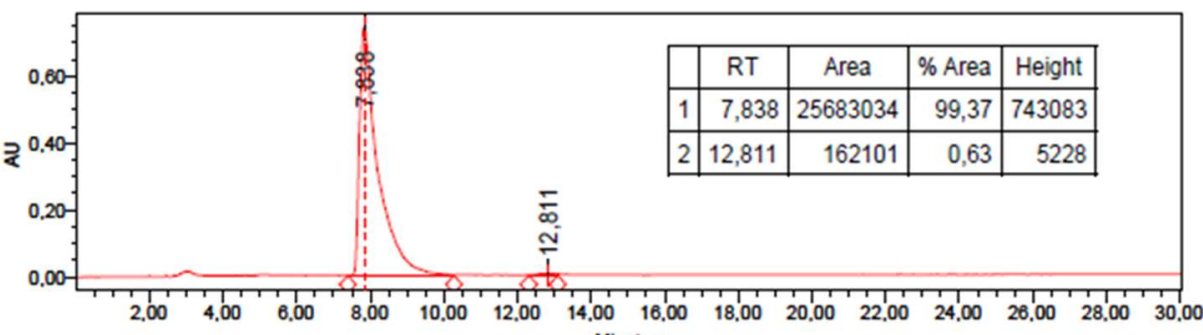
Minutes

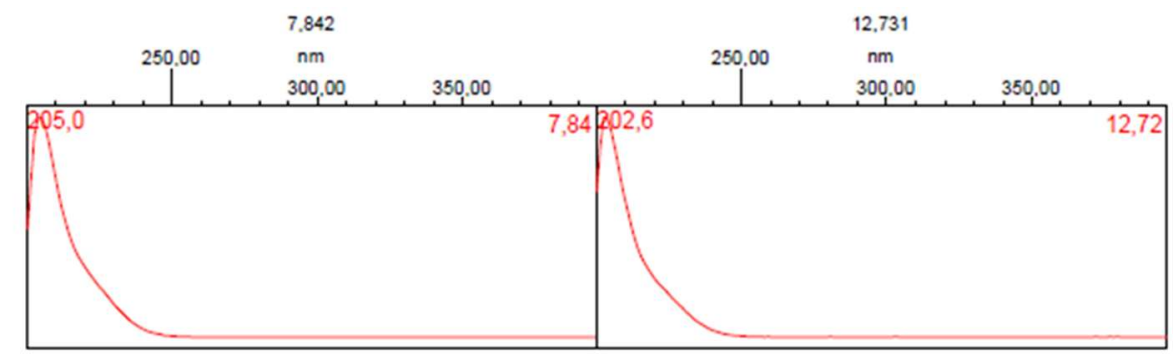

3) Solid : t=45 $\mathrm{min}$.

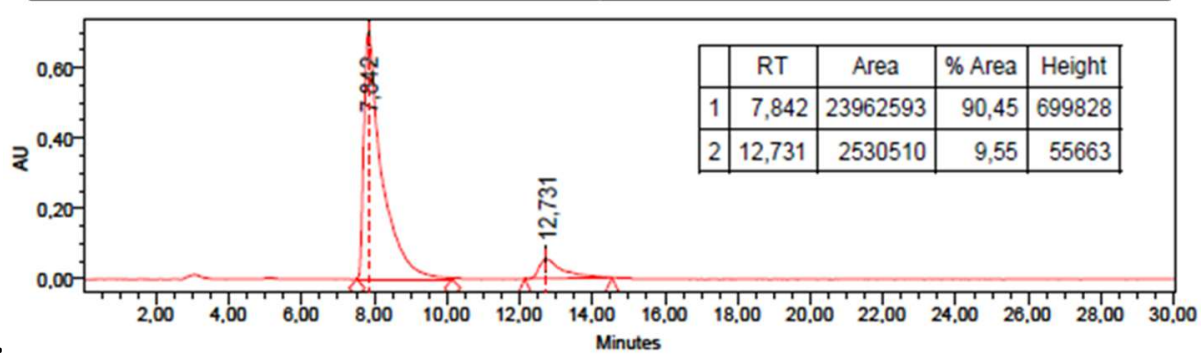




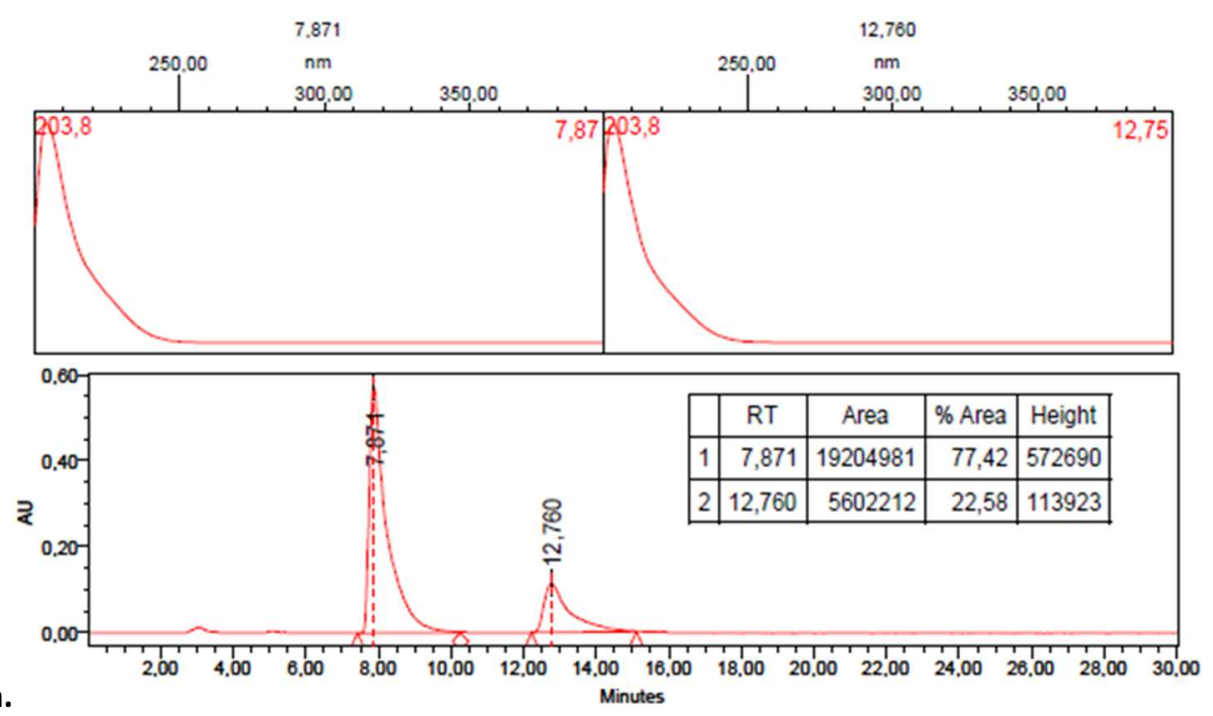

4) Solid : $t=55 \mathrm{~min}$.

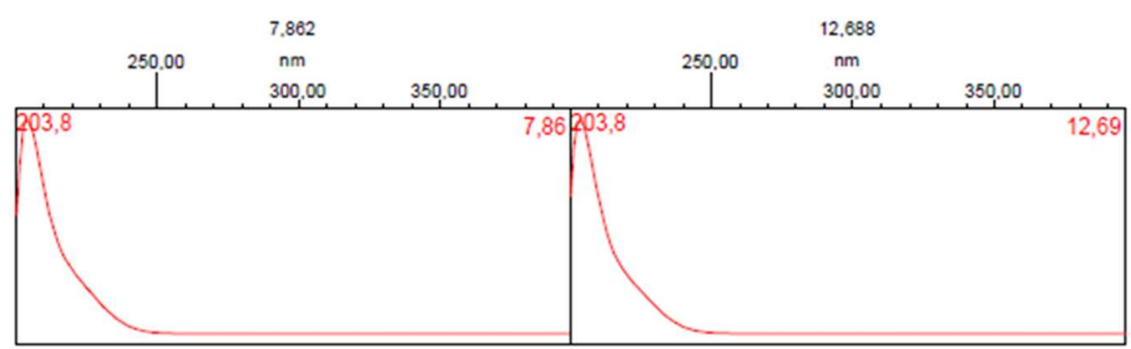

5) Solid : t=65 $\mathrm{min}$.

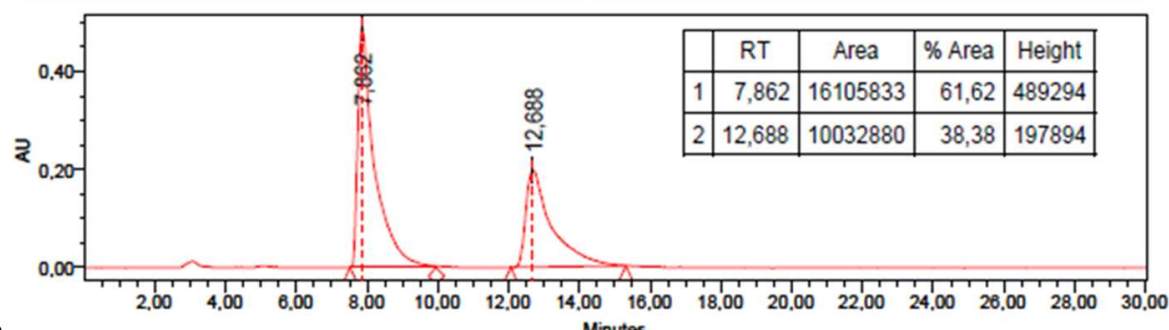

Minutes

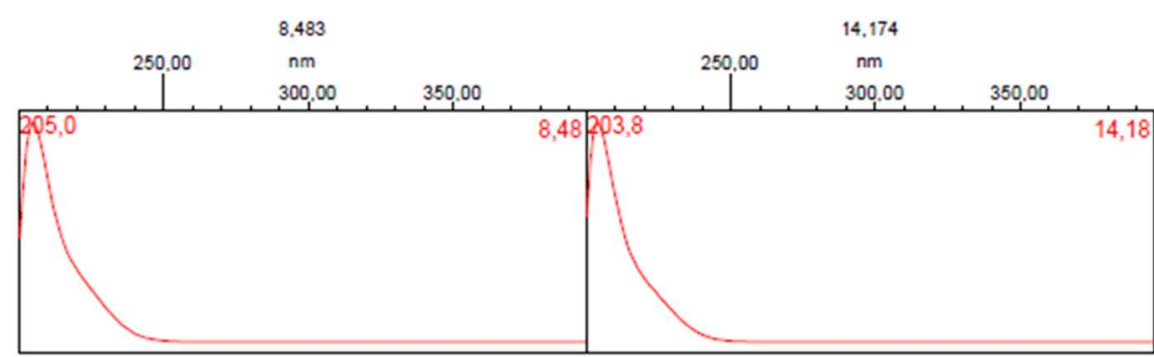

6) Solid : t=75 $\mathrm{min}$.

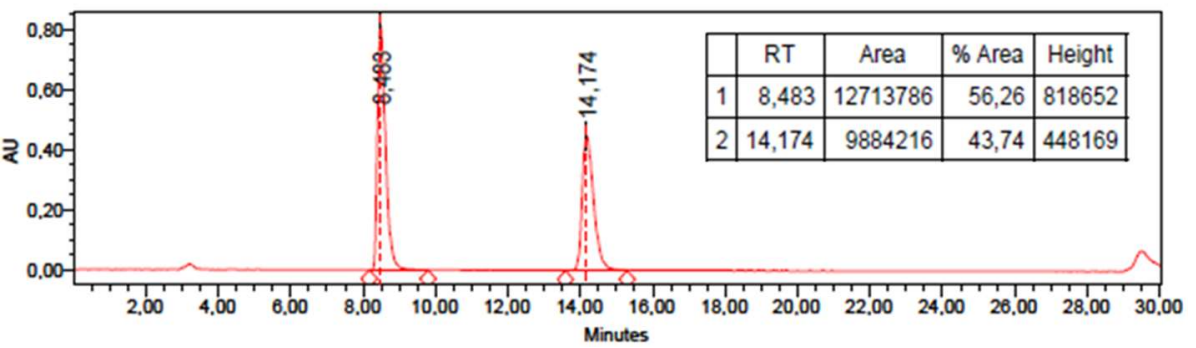




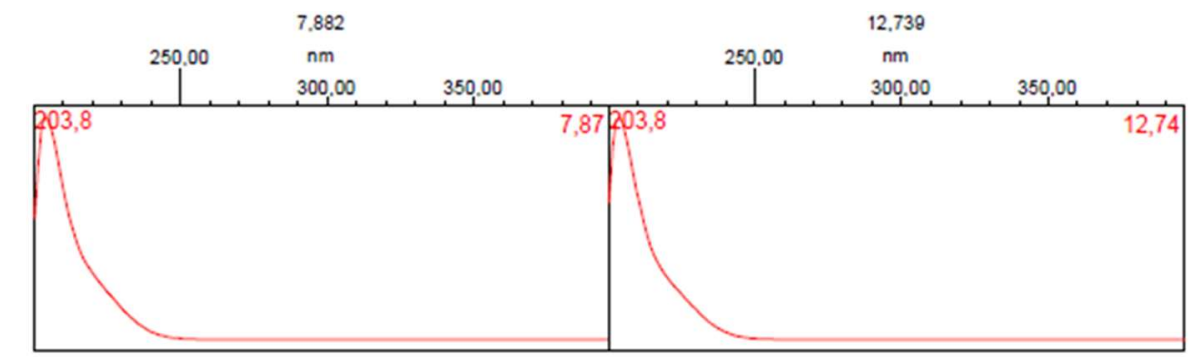

7) Solid : t=85 $\mathrm{min}$.

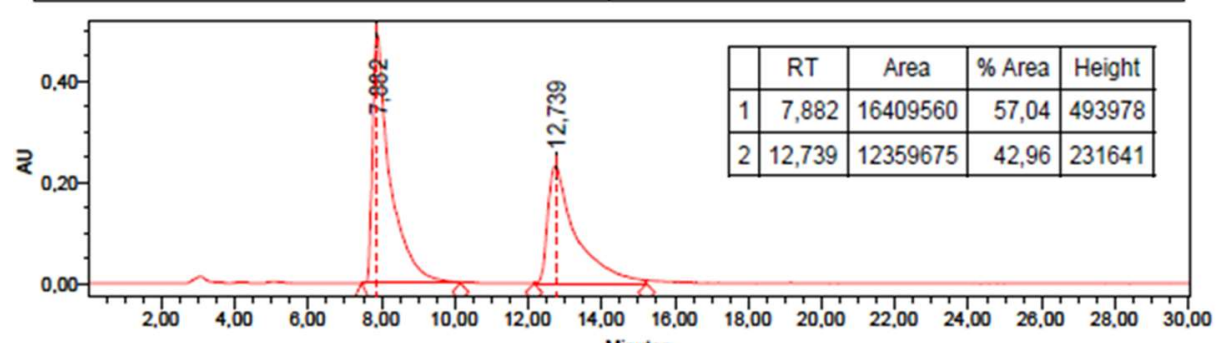
Minutes

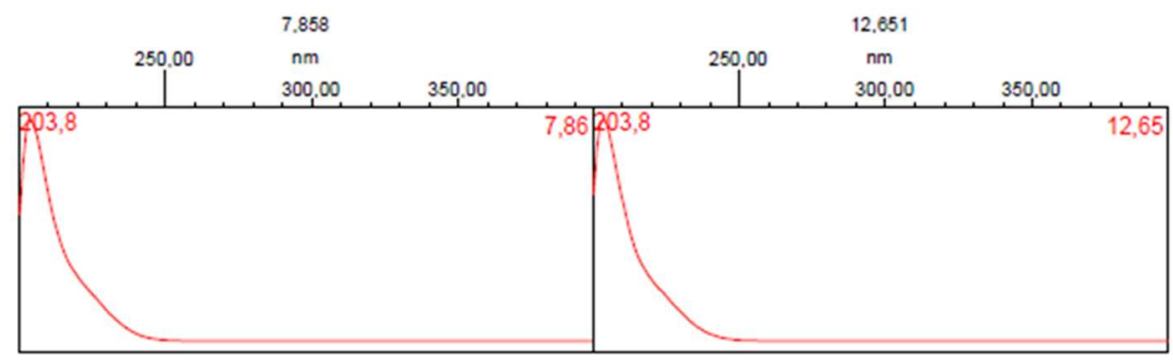

8) Solid : $\mathrm{t}=95 \mathrm{~min}$.
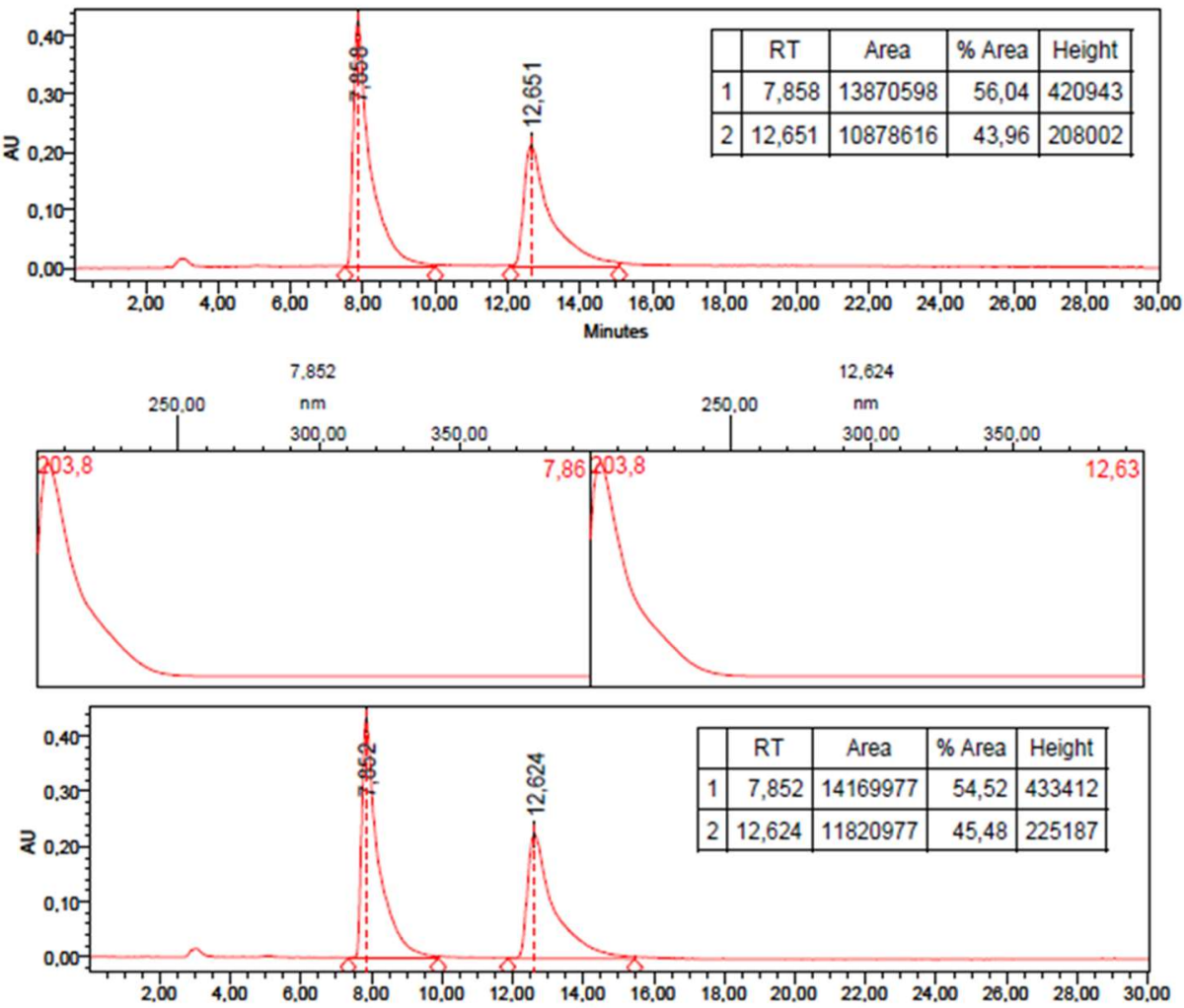

9) Solid : t=105 min. 


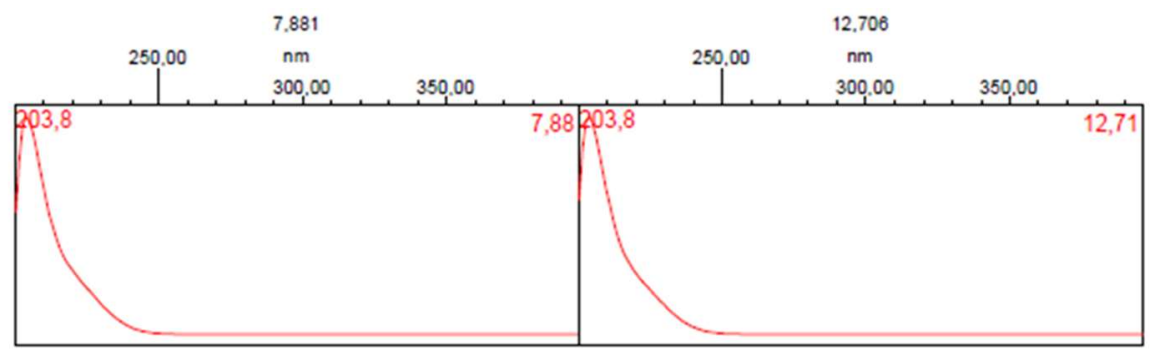

10) Solid : t=115 $\min$
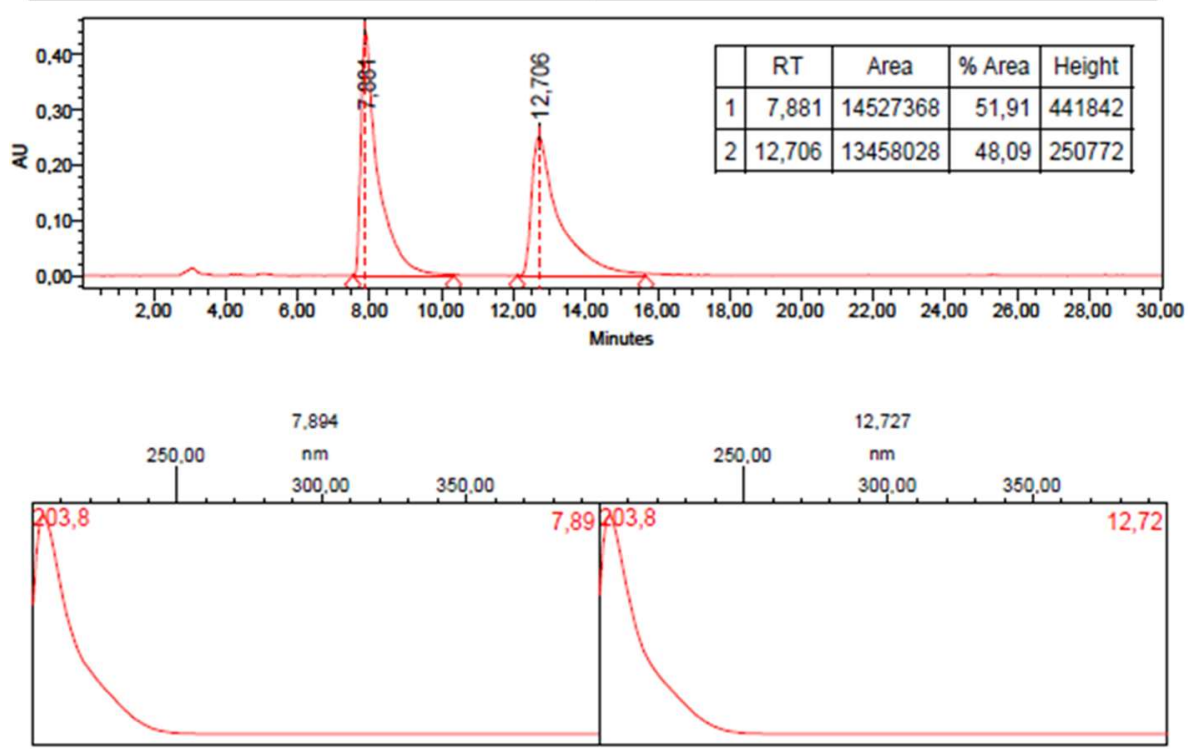

11) Solid : $t=135 \mathrm{~min}$
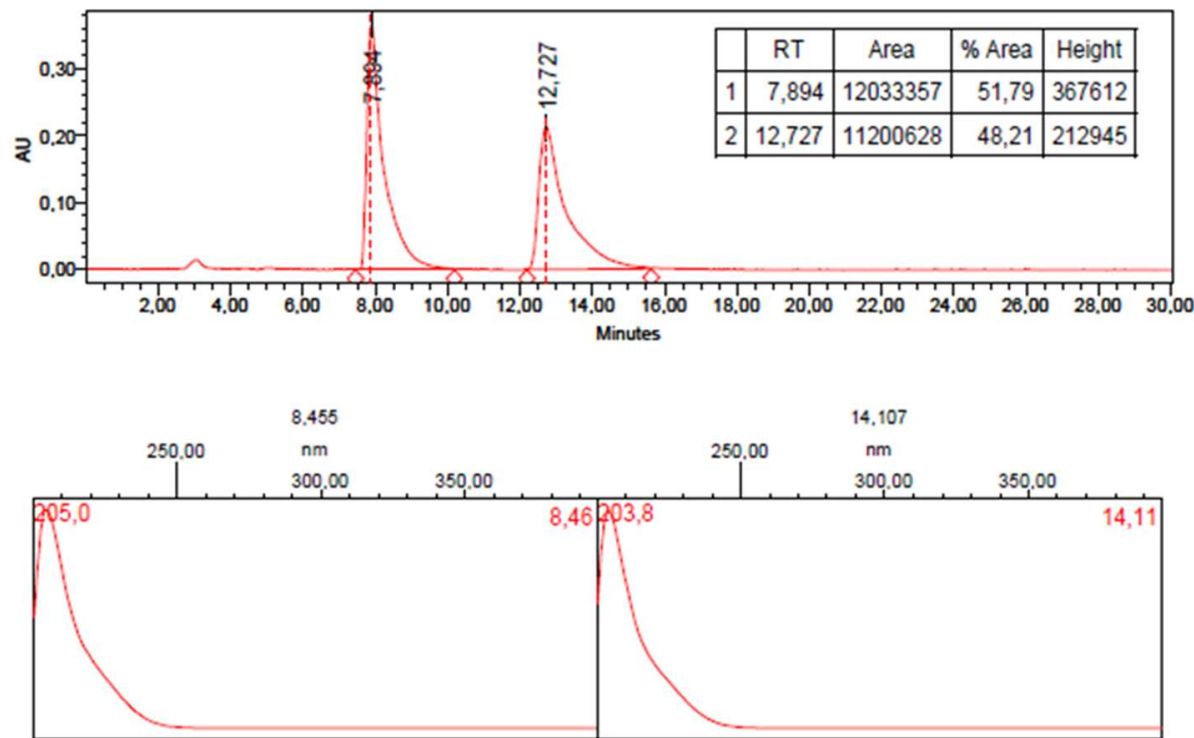

\section{2) Solid : $t=155 \mathrm{~min}$}

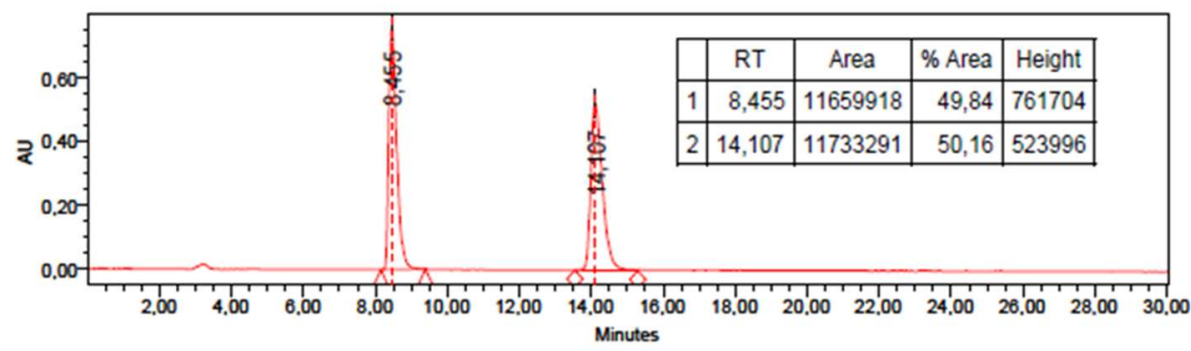

Figure SI-7. UV spectrum index plot and chiral HPLC chromatograms of the solid fractions during the kinetic follow-up. 


\section{Liquid Phase kinetic Follow-up:}

Table SI-7. Liquid Phase kinetic Follow-up

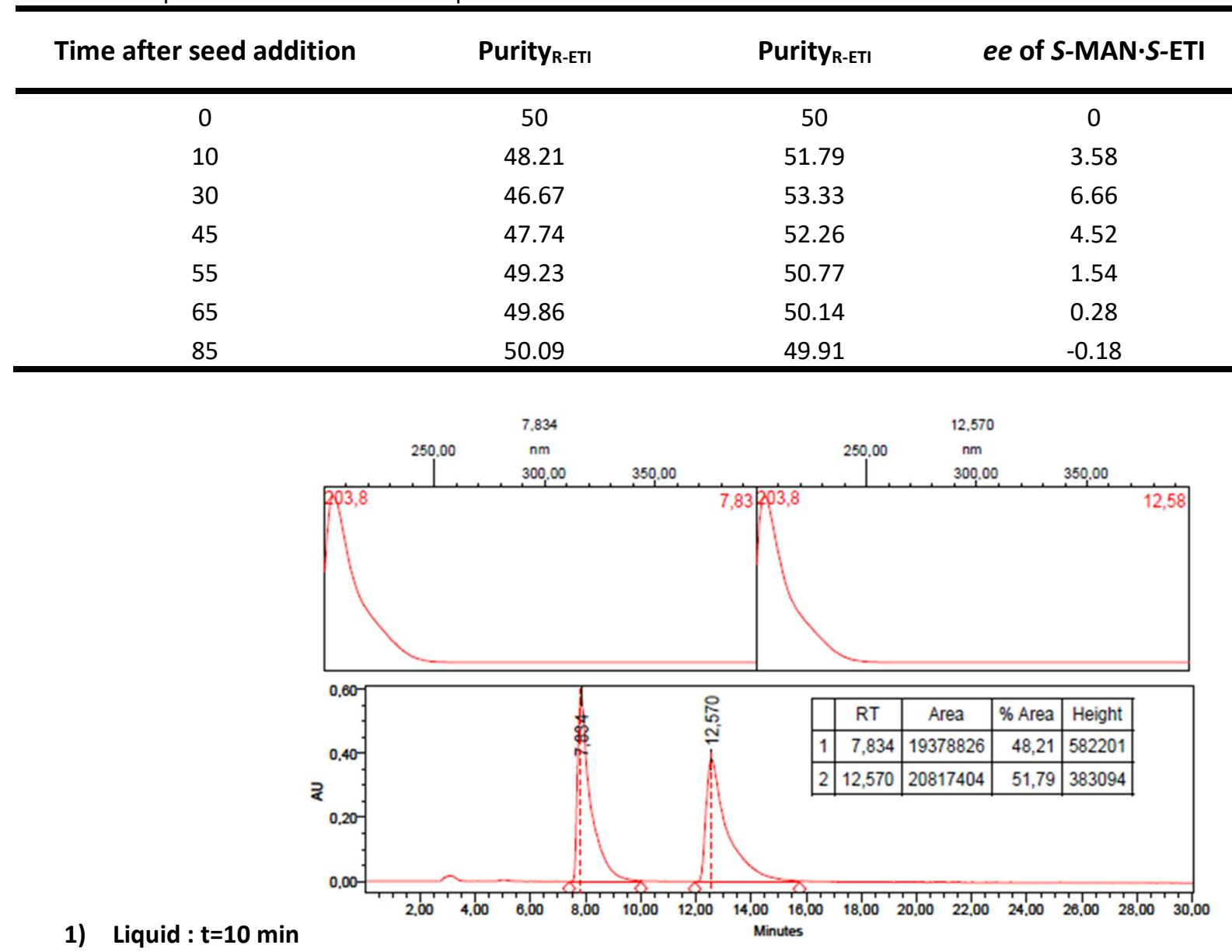

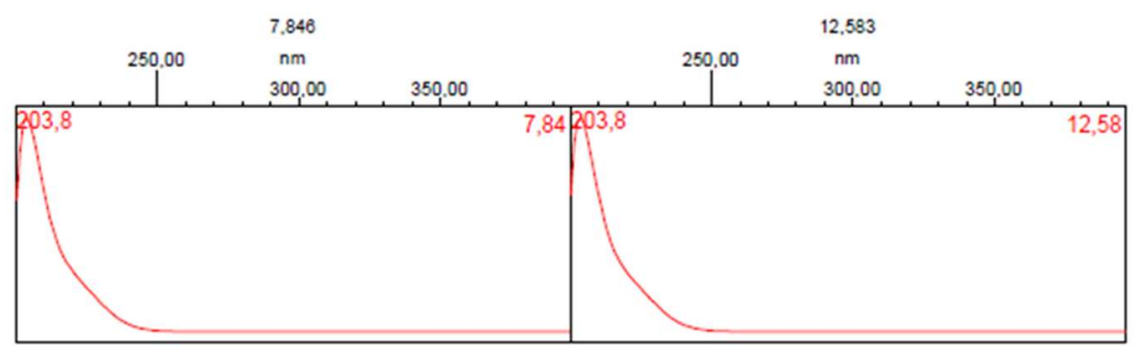

2) Liquid : $t=30 \mathrm{~min}$

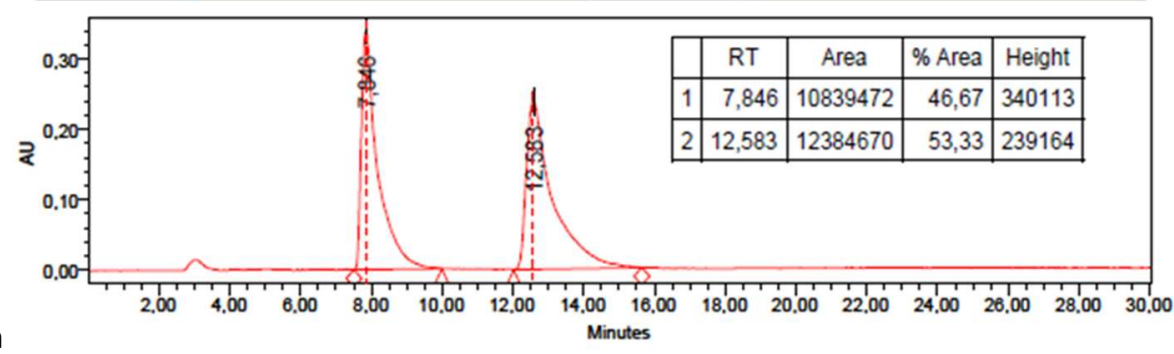


3) Liquid : t=45 $\mathrm{min}$
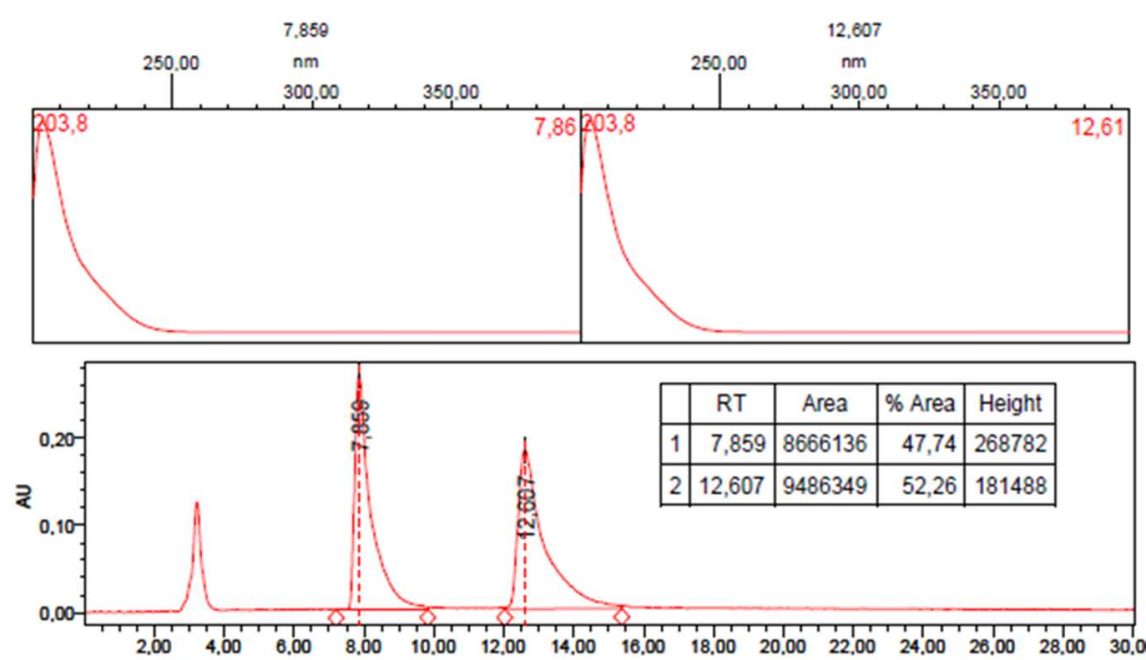
Minutes

4) Liquid : t=55 $\mathrm{min}$
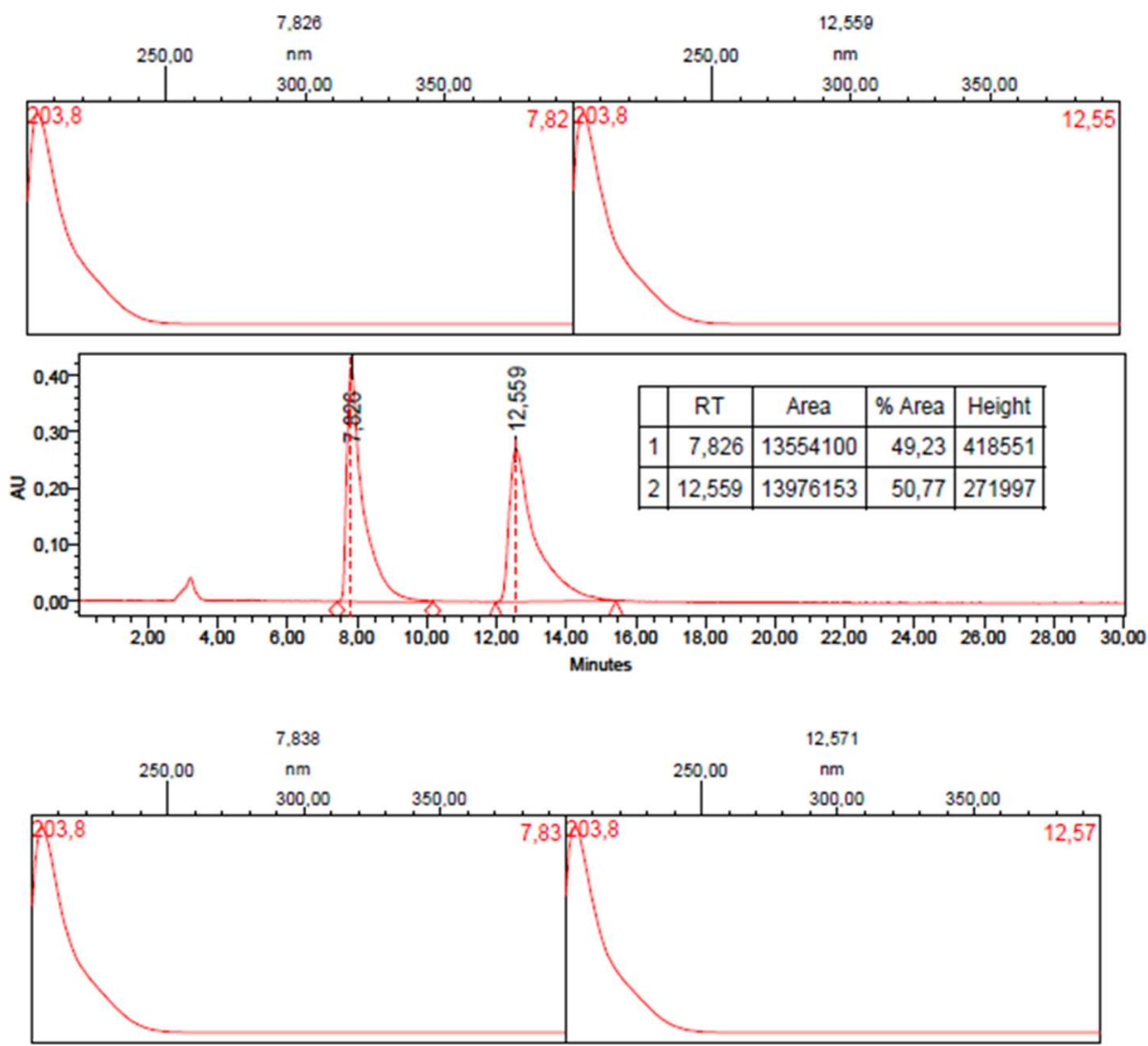

5) Liquid : t=65 $\mathrm{min}$

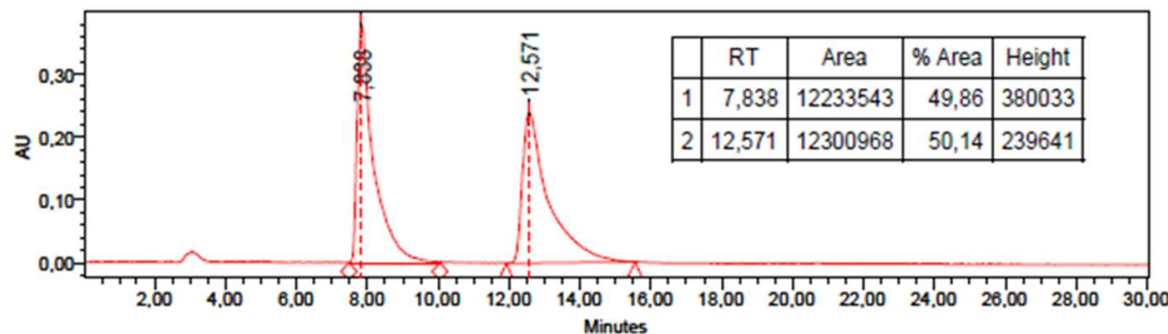



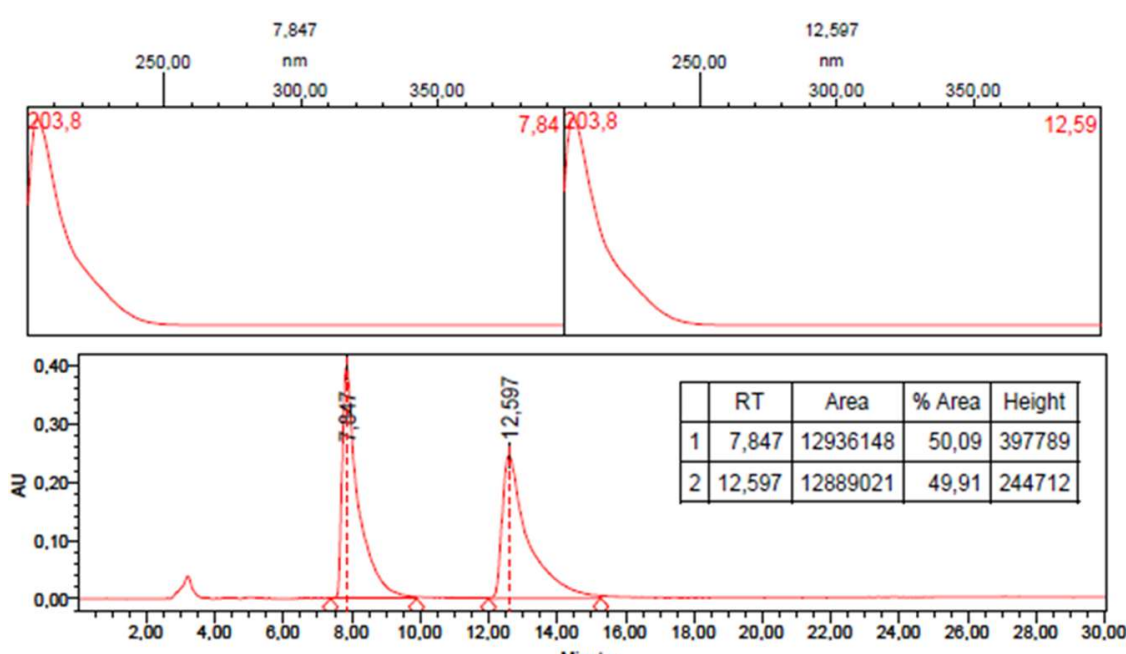

6) Liquid : $t=85 \mathrm{~min}$

Figure SI-8. UV spectrum index plot and chiral HPLC chromatograms of the liquid fractions during the kinetic follow-up. 


\section{Cocrystal dissociation in separate components}

The cocrystal was separated into components following the same procedure as used for the preparation of $R$-etiracetam (see materials section). As an example, $300 \mathrm{mg}$ of S-MAN.S-ETI was suspended in $50 \mathrm{ml}$ of ehtylether for $24 \mathrm{~h}$. The suspension was filtered, and the solid material analyzed by XRPD (Fig. SI-9) and HPLC (SI-12-1), showing pure S-etiracetam. The mother liquor obtained after filtration was left to evaporate. The solid obtained was slurried in $5 \mathrm{ml}$ of dichloromethane for $24 \mathrm{~h}$. The suspension was filtered and the obtained solid analyzed by XRPD (Fig. SI-10 and HPLC (Fig. SI-12-2), showing pure S-mandelic acid.

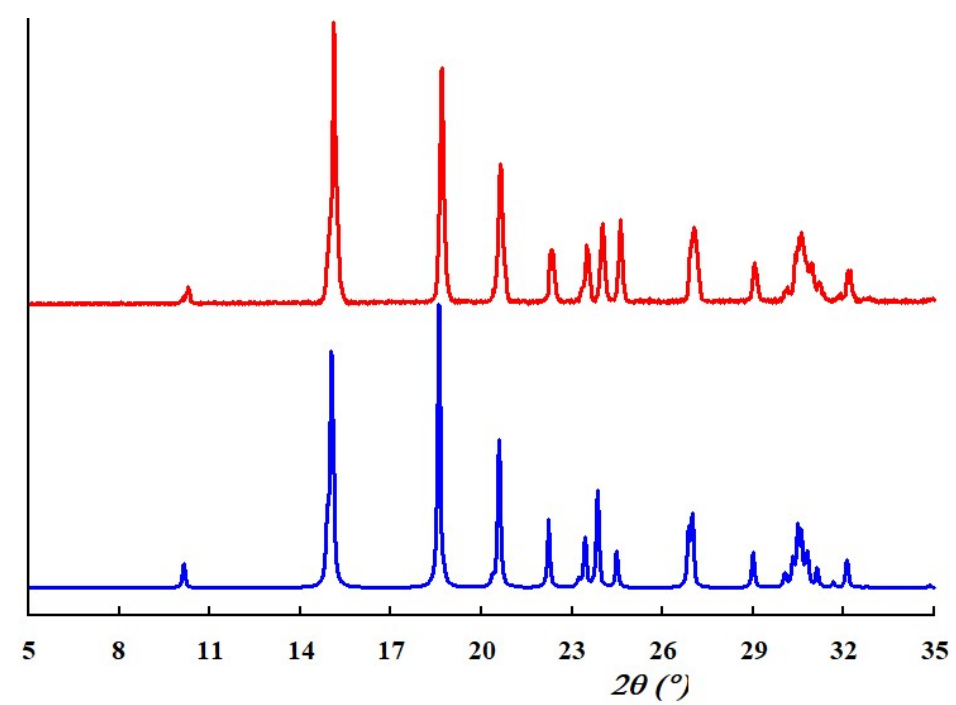

Fig. SI-9. Comparison of the experimental XRPD pattern of S-etiracetam obtained after dissociation of S-MAN.SETI (red) with the calculated of S-etiracetam (blue, CSD refcode OMIVUB ${ }^{[4]}$ ).

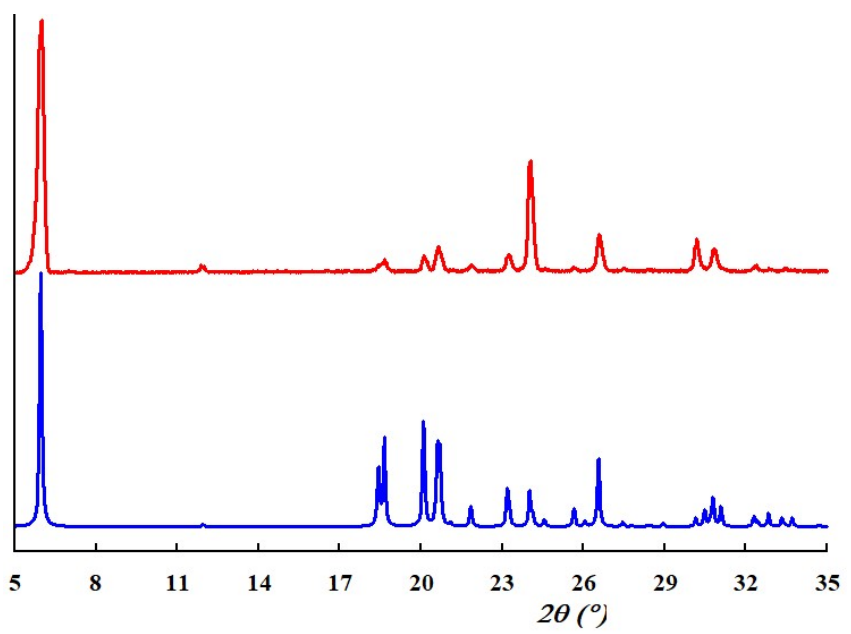

Fig. SI-10. Comparison of the experimental XRPD pattern of $S$-mandelic acid obtained after dissociation of $S$ MAN.S-ETI (red) with the calculated of S-mandelic acid (blue, CSD refcode FEGHAA ${ }^{[5]}$ ).

The initial solid materials was also analyzed by HPLC, showing a 50/50 composition prior to the dissociation process. HPLC of the material obtained upon dissociation of the cocrystal shows full separation of both parent compounds. 


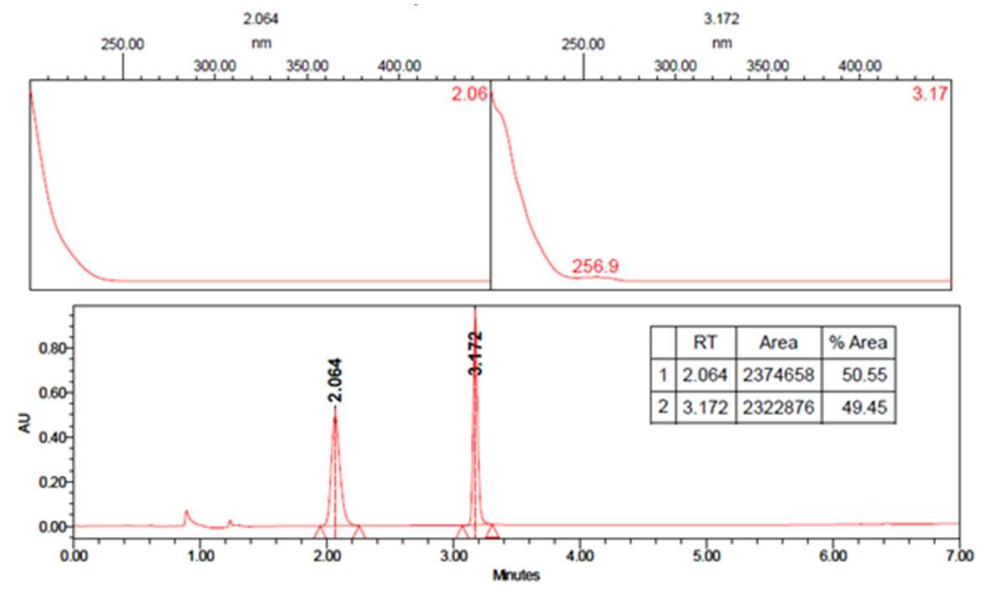

Fig. SI-11. UV spectrum index plot and reverse HPLC chromatograms used to evaluate the initial composition of S-etiracetam (1) vs S-mandelic acid (2) in the cocrystal obtained.

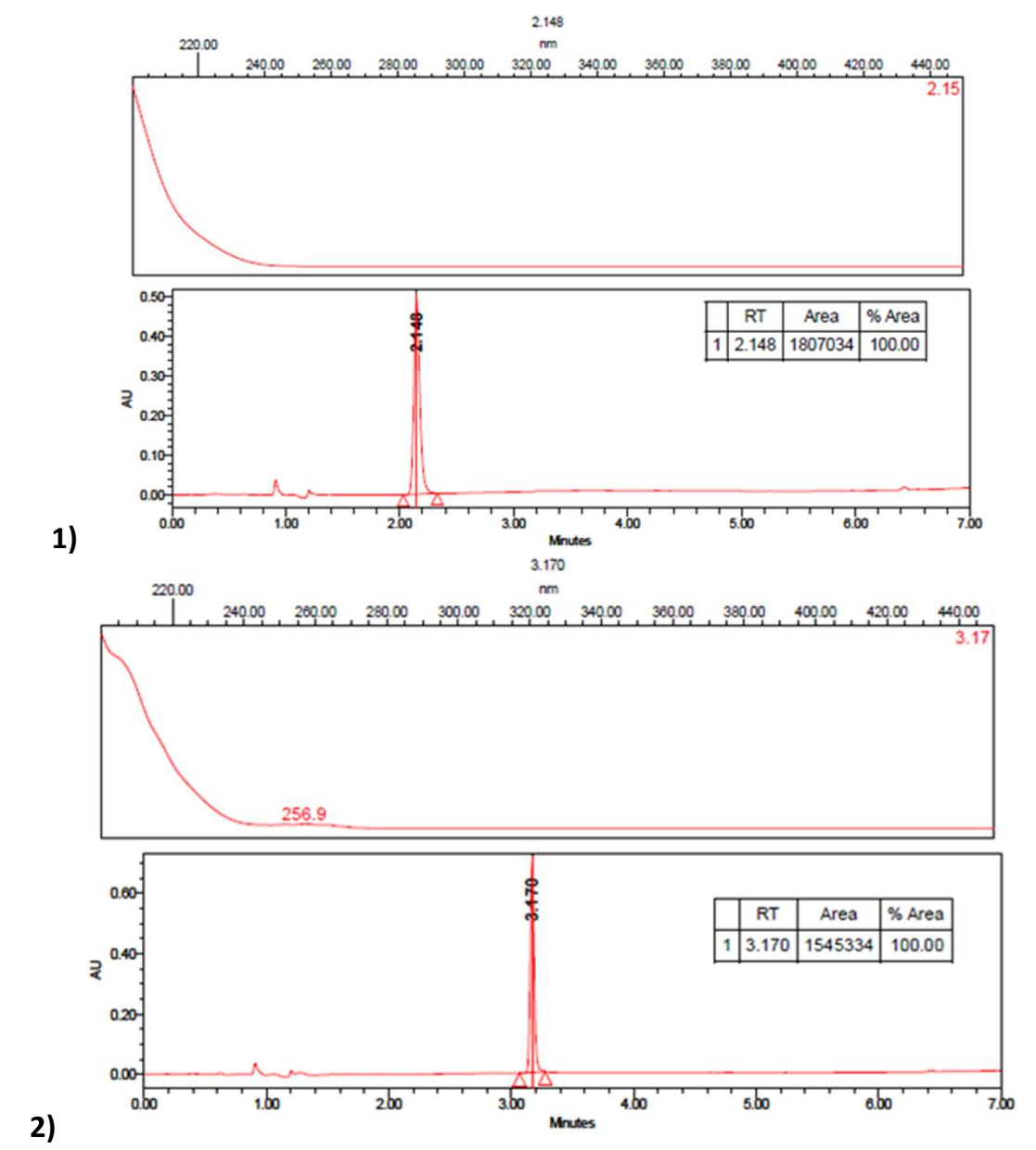

Fig. SI-12. UV spectrum index plot and reverse HPLC chromatograms used to evaluate the purity of S-etiracetam (1) and S-mandelic acid (2) after dissociation of the cocrystal into the parent components. 